YITP-SB-02-50

\title{
Holography and Quaternionic Taub-NUT
}

\author{
Konstantinos Zoubos \\ C. N. Yang Institute for Theoretical Physics \\ State University of New York at Stony Brook \\ Stony Brook, New York 11794-3840 \\ U. S. A. \\ kzoubos@insti.physics.sunysb.edu
}

\begin{abstract}
As a concrete application of the holographic correspondence to manifolds which are only asymptotically Anti-de Sitter, we take a closer look at the quaternionic Taub-NUT space. This is a four dimensional, non-compact, inhomogeneous, riemannian manifold with the interesting property of smoothly interpolating between two symmetric spaces, $\mathrm{AdS}_{4}$ itself and the coset $\mathrm{SU}(2,1) / \mathrm{U}(2)$. Even more interesting is the fact that the scalar curvature of the induced conformal structure at the boundary (corresponding to a squashed three-sphere) changes sign as we interpolate between these two limiting cases. Using twistor methods, we construct the bulk-to-bulk and bulk-to-boundary propagators for conformally coupled scalars on quaternionic Taub-NUT. This may eventually enable us to calculate correlation functions in the dual strongly coupled CFT on a squashed $\mathrm{S}^{3}$ using the standard AdS/CFT prescription.
\end{abstract}




\section{Introduction}

Since the formulation of the AdS/CFT conjecture in [1, 2, 3, there has been considerable effort in trying to check whether its validity extends to situations more general than the original $\operatorname{AdS}_{d+1} \times \mathrm{S}^{D-d-1}$ cases (where $D$ is 10 or 11 ). Assuming one chooses to remain in the limit where classical supergravity is applicable to some extent, there are two obvious generalisations. The first one is to use a different compact internal manifold (or orbifold). Since in this case we preserve the AdS part of the spacetime the dual field theory remains conformal, but substituting internal manifolds of different holonomies leads to less than maximal supersymmetry. Examples of this type are rather numerous and we refer to [4] for an exhaustive list of references.

The other route is to replace the AdS factor of the compactification by a different noncompact Einstein manifold $X_{d+1}$. If $X_{d+1}$ asymptotically gives $\mathrm{AdS}_{d+1}$ as the boundary is approached, then the dual field theory is expected to be conformal only in the UV. (Examples of this are the AdS-Schwarzschild black holes of [2, 5].) Other examples of non-AdS space-time manifolds include (in no particular order) the domain-wall and domain-wall black hole solutions of [6] and 7] respectively, pp-waves in $\operatorname{AdS}$ [8, 9, the coset spaces considered in [10, the de Sitter/CFT correspondence (e.g. [11]), and cases where AdS is modded out by discrete groups, as in 12, 13. Finally, 14] considers the implications for holography when one has manifolds whose boundary conformal structure is degenerate.

There also exists a method, called holographic renormalisation [15, 16] (see [17] for a review), that enables the systematic computation of renormalised correlation functions for operators in the field theory dual to any asymptotically AdS space. (An Einstein manifold with negative cosmological constant is called "asymptotically AdS" if, like AdS itself, the metric has a double pole as the boundary is approached, so that one obtains not a boundary metric, but a conformal class of metrics. See [18 for the precise definition.) This method has been applied to asymptotically AdS domain wall solutions of supergravity that describe RG flows in the dual field theory [19, 20]. Holographic renormalisation depends on a near-boundary analysis, in which the bulk fields are expanded in the radial coordinate transverse to the boundary, and the field equations are solved term-by-term in this expansion. However, this is a local analysis which cannot specify all the coefficients in the series, in particular those that are related to non-local quantities like correlation functions. To do that one would like to have an exact solution of the bulk field equations, extending smoothly to the deep interior of the bulk manifold.

Here we are concerned with the quaternionic Taub-NUT manifold (QTN from now on) which is a four-dimensional riemannian Einstein manifold, a limiting case of which corresponds to $\mathrm{AdS}_{4}$ (or, since we work exclusively with euclidean signature, quaternionic hyperbolic space $\mathbb{H H}^{1}$ ). The Weyl tensor of QTN is self-dual (rather than zero as is the case for hyperbolic space) and so, unlike $\mathbb{H H}^{1}$, it is not conformally flat. However, like $\mathbb{H H}^{1}$, its metric has a double pole as one approaches the boundary, so it is asymptotically AdS. We will consider the Dirichlet problem for a scalar propagating in the fixed QTN background and compute exact bulk-to-bulk and bulk-to-boundary propagators in the conformally coupled case. This may eventually allow us to calculate two- and higher-point functions in the dual theory by applying the methods of holographic renormalisation.

We should note that the QTN manifold has already been used in generalising the AdS/CFT correspondence in 21, 22, 23, 24, but all this previous work has been concerned with calculating Casimir energies for the dual field theory. We believe that our work will take the correspondence one step further. 
As is well known, the boundary of $\mathrm{HH}^{1}$ is the bi-invariant ("round") metric on $\mathrm{S}^{3}$ :

$$
\mathrm{d} s^{2}=\sigma_{1}^{2}+\sigma_{2}^{2}+\sigma_{3}^{2} .
$$

This is of course a particular choice of representative for the conformal structure of the boundary. Since this metric is conformally flat, by a different choice of representative one usually writes the boundary as three-dimensional flat space $\mathbb{R}^{3}$ and, via AdS/CFT, proceeds to define a dual conformal field theory thought of as living on this flat boundary.

In [25], Pedersen applied earlier ideas of LeBrun [26] to solve the problem of obtaining an Einstein 4-metric that has as conformal infinity the Berger sphere, i.e. the $\mathrm{SU}(2) \times \mathrm{U}(1)-$ invariant "squashed" three-sphere metric" :

$$
\mathrm{d} s^{2}=\sigma_{1}^{2}+\sigma_{2}^{2}+\lambda \sigma_{3}^{2} .
$$

The resulting complete Einstein manifold with negative cosmological constant turns out to be quaternionic Taub-NUT. Since the metric on QTN smoothly extends across the boundary to the conformal structure represented by (1.2), it is natural to expect that one can apply the AdS/CFT prescription to this case. We show that one can find (scalar) bulk-to-bulk and bulkto-boundary propagators that generalise the ones known for $\mathbb{H H}^{1}$ and thus in principle obtain correlation functions for certain operators in the dual $(3+0)$ conformal field theory living on the squashed three-sphere. However, since the squashed three-sphere is not conformally flat, we will be forced to consider the dual CFT as living on a curved background. (Examples of AdS/CFT with curved boundaries can be found in e.g. [29, 9, 30, 31].)

We do not expect that we might learn something about realistic field theories in this fashion. For a start, we discuss only riemannian metrics and it is not clear whether one can translate these results to the lorentzian regime. Also, we make no specific reference to any M-theory or string compactifications, although one could directly replace $\mathbb{H H}^{1}$ by QTN in the standard Freund-Rubin ansatz to obtain e.g. a $\mathrm{QTN} \times \mathrm{S}^{7}$ vacuum solution of 11 -dimensional (euclidean) supergravity. We will also not touch the issue of stability, at least from the gravity side of the correspondence (but see below).

The point of view we take is that we might learn something about holography by examining a more general setting that is still tractable (because of the quaternionic geometry). In particular we note the following interesting features of holography on QTN that provided motivation for this work and, we believe, merit further investigation:

1. Obviously the round (conformally flat) metric (1.1) on $\mathrm{S}^{3}(\lambda=1)$ has positive scalar curvature. However as we squash the three-sphere by increasing the parameter $\lambda$, we reach a point $(\lambda=4)$ where the scalar curvature becomes zero, and for $\lambda>4$ it becomes negative (this is discussed e.g. in [32, p. 38). It is well documented in the AdS/CFT literature (e.g. in $[33,3]^{2}$ ) that for the field theory dual to any negative curvature Einstein Manifold to be stable, it is necessary that the induced boundary manifold have positive scalar curvature. So it is nice to be able to explore a case where we can smoothly move

\footnotetext{
${ }^{1}$ Pedersen's work can be thought of as an example of the subsequent theorem of Graham and Lee 27 that the conformal class of a given metric on $\mathrm{S}^{d}$, sufficiently close to the round one, can arise as the conformal infinity of a suitable $d+1$-dimensional metric on the ball $B^{d+1}$. (The general problem of whether such metrics exist was posed in 28.)

${ }^{2}$ See also 35$]$.
} 
from a positive to a negative scalar curvature boundary metric, and thus try to understand the apparent instability of the boundary theory in bulk terms. (Presumably the instability will have something to do with emission of a "large" brane as in [33.) The point $\lambda=4$ where the boundary has zero scalar curvature is also quite intriguing in its own right.

2. If we take our parameter $\lambda$ to its limiting value of infinity, the Berger sphere degenerates and we end up with an effectively two dimensional theory. In [10] it is claimed that the resulting CFT is in fact well-behaved (being dual to a stable M-theory compactification on $\left.\mathrm{SU}(2,1) / \mathrm{U}(2) \times S^{7}\right)$. It is thus interesting to analyse how the transition from a $3 \mathrm{~d}$ to a $2 \mathrm{~d}$ CFT is made, and how the apparent stability can be reconciled with the point made above, since we are in the negative boundary scalar curvature regime (in fact, the scalar curvature is negatively infinite $)^{3}$.

Our approach to calculating propagators is inspired by Page's construction of Green's functions for massless scalars propagating on gravitational multi-instantons [36. This work is essentially a generalisation of those results to the non-Ricci-flat case. In the next section we give an overview of the quaternionic Taub-NUT manifold. In section 3 we specialise to the metric we will use for QTN, which is the one found by Pedersen, and exhibit several interesting limiting cases. In section 4 we review known results for Green's functions for these cases. The construction of the conformally coupled Green's function for the Pedersen metric follows in sections $5,6,7$. In section 8 we briefly discuss the boundary theory and we conclude with various open issues in section 9 .

\section{Some facts about Quaternionic Taub-NUT}

In $4 n$ real dimensions, we use the term quaternionic Taub-NUT $\left(\mathrm{QTN}_{n}\right)$ for the noncompact inhomogeneous quaternionic Kähler manifold that has euclidean Taub-NUT as its hyperkähler limit. A quaternionic Kähler manifold is defined (e.g. [37, 38]) to be a $4 n$-dimensional oriented riemannian manifold with holonomy contained in $\operatorname{Sp}(n) \cdot \operatorname{Sp}(1)$ (which means $\left.(\operatorname{Sp}(n) \times \operatorname{Sp}(1)) / \mathbb{Z}_{2}\right)$. In four dimensions (which is all we consider here) the holonomy requirement $\operatorname{Sp}(1) \cdot \mathrm{Sp}(1) \cong \mathrm{SO}(4)$ is vacuous, so one defines quaternionic Kähler manifolds as those Einstein manifolds whose Weyl tensor is self-dual (or anti-self-dual). Since we consider only the four dimensional case, we write just QTN for $\mathrm{QTN}_{1}$.

Quaternionic Kähler manifolds have (positive or negative) cosmological constant $\Lambda$, and reduce to Ricci-flat hyperkähler manifolds in the limit where $\Lambda$ is taken to zero. We are interested in noncompact manifolds, which (by Myers' theorem) means $\Lambda$ has to be non-positive. In four dimensions there are only two noncompact homogeneous quaternionic Kähler spaces (which, actually, are also symmetric): Hyperbolic space and the noncompact version of $\mathbb{C P}^{2}$, which we denote by $\widetilde{\mathbb{C P}^{2}}$. Being homogeneous, they are coset spaces:

$$
\mathbb{H H}^{1}=\frac{\mathrm{SO}(1,4)}{\mathrm{SO}(4)} \quad \text { and } \quad \widetilde{\mathrm{CP}^{2}}=\frac{\mathrm{SU}(2,1)}{\mathrm{U}(2)} .
$$

As we will see explicitly in the next section, QTN smoothly interpolates between these two

\footnotetext{
${ }^{3}$ This issue has been raised in 14 .
} 
manifolds ${ }^{4}$. Note that there is no analogous interpolation via a smooth manifold between the compact versions of these homogeneous spaces, i.e. $\mathrm{S}^{4}$ and $\mathbb{C P}^{2}$.

We have already mentioned one way to derive QTN, as the "filling in" manifold of the Berger sphere (1.2). QTN can also be seen to be a special case of the (Anti-) de Sitter-Taub-NUT/Bolt family of metrics that appears e.g. in [40. In the notation of 22 this more general metric is

$$
\mathrm{d} s^{2}=V(r)(\mathrm{d} \tau+2 n \cos \theta \mathrm{d} \varphi)^{2}+\frac{\mathrm{d} r^{2}}{V(r)}+\left(r^{2}-n^{2}\right)\left(\mathrm{d} \theta^{2}+\sin ^{2} \theta \mathrm{d} \varphi^{2}\right)
$$

with $V(r)=\left[\left(r^{2}+n^{2}\right)^{2}-2 M r+k^{2}\left(r^{4}-6 n^{2} r^{2}-3 n^{4}\right)\right] /\left(r^{2}-n^{2}\right)$. Apart from the cosmological constant $\left(\Lambda=-3 k^{2}\right)$ there are two parameters, $M$ and $n$, which correspond to a "mass" and "nut charge" respectively. As explained thoroughly in [22, to obtain a nut, i.e. a regular zero-dimensional fixed point set, we require that $V(r=n)=0$, so $M$ and $n$ are related by

$$
M=n-4 n^{3} k^{2} .
$$

A different possible choice for $M$ would lead to a regular two-dimensional fixed point set (a "bolt"). It is the special case of (2.3) (thus the solution without bolts) that gives the QTN manifold. (The precise relation to the metric found by Pedersen is given in appendix A.)

QTN has also been derived by Galicki 41, 42] using the quaternionic Kähler quotient (described in detail in [43, 44, 45]) by considering a non-compact $\mathbb{R}$ action on the 8-dimensional quaternionic projective ball $\mathbb{H H}^{2}=P\left(\mathbb{H}^{1,1} \times \mathbb{H}^{1}\right)$. Finally, it has also been constructed by Ivanov and Valent [46] using harmonic superspace methods [47, 48. (Actually the term "quaternionic Taub-NUT" seems to have first appeared in [46].)

One application of QTN in the literature has been as the sigma-model manifold for scalars coupled to $\mathcal{N}=2$ supergravity. (Scalar couplings in $\mathcal{N}=2$ supergravity are known to be parametrised by negative cosmological constant quaternionic Kähler manifolds [49.) It is in this context that QTN (and its higher dimensional generalisations) was derived by Galicki. More recently, Behrndt and Dall'Agata [50] considered abelian gaugings of its isometries and found domain wall solutions for $d=5, \mathcal{N}=2$ supergravity.

As we mentioned, QTN has already found some uses in an AdS/CFT context ([21, 22, 23, 24]). In [21, 22] it was used as a background for the calculation of the gravitational action and entropy for the AdS-Taub-Bolt manifold that has the same behaviour at infinity. In [23] and [24] the action and entropy of AdS-Taub-NUT were calculated in an intrinsic way using the counterterm subtraction method of [15, 51]. The result is somewhat unsettling in that the gravitational entropy turns out to be negative, which is probably linked to problems with the lorentzian interpretation [14. Perhaps learning more about the dual CFT will help to understand this issue better.

\footnotetext{
${ }^{4}$ By "interpolation" we mean that there is a continuous range of QTN metrics (labelled as we will see by the "nut charge"), two specific examples of which correspond to $\mathbb{H H}^{1}$ and $\widetilde{\mathrm{CP}^{2}}$. So this is different from RG flows in AdS/CFT (see e.g. 39] for orientation), where one interpolates between different solutions of some gauged supergravity (usually corresponding to UV and IR regimes in the boundary theory) by varying the radial parameter which is interpreted as an energy scale. However, as we will see in section 8, one could perhaps consider the deformation of the boundary metric as a sort of marginal flow.
} 


\section{The Pedersen Metric for Quaternionic Taub-NUT}

The metric we will use to describe the quaternionic Taub-NUT manifold is a slight rewriting of the one first written down by Pedersen [25. Actually, in [25] there are two metrics, which correspond to an oblate and prolate squashing of the boundary sphere ${ }^{5}$. We will treat them in turn.

\subsection{The Oblate case}

The form of the Pedersen metric that we will use is:

$$
g_{\mu \nu}^{(m, k)}: \quad \mathrm{d} s^{2}=\frac{4}{\left(1-k^{2} r^{2}\right)^{2}}\left[\frac{1+m^{2} r^{2}}{1+m^{2} k^{2} r^{4}} \mathrm{~d} r^{2}+r^{2}\left(1+m^{2} r^{2}\right)\left(\sigma_{1}^{2}+\sigma_{2}^{2}\right)+\frac{r^{2}\left(1+m^{2} k^{2} r^{4}\right)}{1+m^{2} r^{2}} \sigma_{3}^{2}\right] .
$$

Here $\sigma_{1}, \sigma_{2}, \sigma_{3}$ are the usual $\mathrm{SU}(2)$ one-forms, defined by

$$
\begin{aligned}
\sigma_{1} & =\frac{1}{2}(\cos \psi \mathrm{d} \theta+\sin \psi \sin \theta \mathrm{d} \varphi), \\
\sigma_{2} & =\frac{1}{2}(-\sin \psi \mathrm{d} \theta+\cos \psi \sin \theta \mathrm{d} \varphi), \\
\sigma_{3} & =\frac{1}{2}(\mathrm{~d} \psi+\cos \theta \mathrm{d} \varphi) .
\end{aligned}
$$

(where $0 \leq \theta \leq \pi, 0 \leq \varphi \leq 2 \pi, 0 \leq \psi \leq 4 \pi$ ). With this convention they satisfy the cyclic relation:

$$
\mathrm{d} \sigma_{1}=-2 \sigma_{2} \wedge \sigma_{3}, \text { etc. }
$$

We have modified the expression in 25] by explicitly including the cosmological constant, which can be seen to be $\Lambda=-3 k^{2}$. The parameter $m$ will soon be seen to correspond to the nut charge. So the metric depends on two (real) parameters, $m$ and $k$, both with dimension $[L]^{-1}$, which can be varied independently. We can restrict to positive values of $m$ and $k$ without loss of generality [52. The scalar curvature can be seen to be $\mathcal{R}=-12 k^{2}$. One can also check that the Weyl tensor is anti-self-dual ${ }^{6}$. The isometry group is $\mathrm{SU}(2) \times \mathrm{U}(1)$ for generic values of $m, k$.

This metric is complete within the ball $r<1 / k$ for all values of $m[52]^{7}$. We will thus restrict $r$ to lie within the ball, with the boundary given by $r \rightarrow 1 / k$. There the metric blows up, inducing the conformal structure:

$$
\sigma_{1}^{2}+\sigma_{2}^{2}+\frac{1}{1+m^{2} / k^{2}} \sigma_{3}^{2}
$$

So this corresponds to an oblate squashing of the three sphere, since the parameter $\lambda$ in (1.2) is less than one.

\footnotetext{
${ }^{5}$ We recall that "oblate squashing" corresponds to flattening the sphere at the poles, while "prolate squashing" means elongating the sphere at the poles.

${ }^{6}$ In [25] the Weyl tensor turns out to be self-dual due to a different convention for the $\operatorname{sigmas}\left(\mathrm{d} \sigma_{i}=\sum \epsilon_{i j k} \sigma_{j} \wedge\right.$ $\left.\sigma_{k}\right)$.

${ }^{7}$ The singularities of quaternionic Taub-NUT in various parameter ranges have also been considered in [50].
} 
The $k=0$ limit: Euclidean Taub-NUT Taking the cosmological constant $\Lambda=-3 k^{2}$ to zero, while keeping the nut charge nonzero, we obtain a Ricci-flat metric

$$
g_{\mu \nu}^{(m, k=0)}: \quad \mathrm{d} s^{2}=\left(1+m^{2} r^{2}\right)\left[\mathrm{d} r^{2}+r^{2}\left(\sigma_{1}^{2}+\sigma_{2}^{2}\right)\right]+\frac{r^{2}}{1+m^{2} r^{2}} \sigma_{3}^{2}
$$

which is the metric on the euclidean Taub-NUT ALF space, in the form suitable for generalisation to the multi-Taub-NUT metrics [53, 40. We see that $m$ is indeed related to the nut charge (see appendix A for the precise relation).

\subsection{The Prolate case}

In the metric (3.1) we assumed $m^{2}>0$, but it turns out that this restriction is unnecessary [25]. Indeed, we can analytically continue the parameter $m$ as follows ${ }^{8}$ :

$$
m \rightarrow i \mu \text {. }
$$

In this way we obtain the metric:

$$
g_{\mu \nu}^{(\mu, k)}: \quad \mathrm{d} s^{2}=\frac{4}{\left(1-k^{2} r^{2}\right)^{2}}\left[\frac{1-\mu^{2} r^{2}}{1-\mu^{2} k^{2} r^{4}} \mathrm{~d} r^{2}+r^{2}\left(1-\mu^{2} r^{2}\right)\left(\sigma_{1}^{2}+\sigma_{2}^{2}\right)+\frac{r^{2}\left(1-\mu^{2} k^{2} r^{4}\right)}{1-\mu^{2} r^{2}} \sigma_{3}^{2}\right] .
$$

This metric is complete within the ball $r<1 / k$ as long as the range of $\mu$ is $(0, k)$ [52. For $\mu>k$ the metric develops a true singularity, as can be seen e.g. by the calculation of curvature invariants:

$$
\mathcal{R}^{\mu \nu \kappa \lambda} \mathcal{R}_{\mu \nu \kappa \lambda}=24+24 \frac{\left(1-k^{2} r^{2}\right)^{2}}{\left(1-\mu^{2} r^{2}\right)^{2}} .
$$

Accordingly, we will from now on restrict $\mu$ to lie in the range $(0, k)$, and again $r$ to lie within the ball. As for the induced conformal structure at the boundary $(r=1 / k)$, we find

$$
\sigma_{1}^{2}+\sigma_{2}^{2}+\frac{1}{1-\mu^{2} / k^{2}} \sigma_{3}^{2}
$$

This gives a prolate squashing of the three-sphere, since now $\lambda$ in (1.2) is greater than one.

The boundary metric By a choice of conformal scale, we can define a metric on the boundary that represents the conformal structure (3.9). One possible choice is:

$$
h_{i j}^{(\mu, k)}: \quad \mathrm{d} s^{2}=\left(1-\mu^{2} / k^{2}\right)\left(\sigma_{1}^{2}+\sigma_{2}^{2}\right)+\sigma_{3}^{2}
$$

Here $i, j=1,2,3$. Recall that the value $\mu=0$ corresponds to the round $(\mathrm{SU}(2) \times \mathrm{SU}(2)$ invariant $)$ metric on $\mathrm{S}^{3}$. Away from that value, the isometry group is $\mathrm{SU}(2) \times \mathrm{U}(1)$, the same as the bulk manifold. The scalar curvature of $h_{i j}^{(\mu, k)}$ is found to be:

$$
\mathcal{R}^{(3)}=\frac{3 k^{2}-4 \mu^{2}}{2\left(1-\mu^{2} / k^{2}\right)^{2}}
$$

As mentioned in the introduction, the scalar curvature becomes zero and then negative as we increase $\mu^{2}$ from zero to its limiting value of $k^{2}$. For the value $\mu^{2}=\frac{3}{4} k^{2}$ we obtain $\mathcal{R}^{(3)}=0$. As we approach $k^{2}$ the (negative) scalar curvature blows up, indicating that the boundary metric becomes degenerate.

Returning to (3.7), we note two limiting cases of special interest.

\footnotetext{
${ }^{8}$ There is a deeper meaning to this analytic continuation which will become clear in section (5.1).
} 
The $\mu=0$ limit: Euclidean $\mathbf{A d S}_{4}\left(\mathbb{H H}^{1}\right)$ Taking $\mu=0$ in (3.7) we obtain

$$
g_{\mu \nu}^{(\mu=0, k)}: \mathrm{d} s^{2}=\frac{4}{\left(1-k^{2} r^{2}\right)^{2}}\left[\mathrm{~d} r^{2}+r^{2}\left(\sigma_{1}^{2}+\sigma_{2}^{2}+\sigma_{3}^{2}\right)\right]
$$

which is clearly the metric on $\mathbb{H H}^{1}$ in polar coordinates. (See appendix $\mathrm{B}$ for the relation to the more commonly used upper half plane metric on $\mathbb{H H}^{1}$.) The isometry group is now enhanced to $\mathrm{SO}(1,4)$, which will become the conformal group of the boundary. As discussed, the boundary of $\mathrm{HH}^{1}$ is simply $\mathrm{S}^{3}$ with the round metric,

$$
h_{i j}^{(0, k)}=\sigma_{1}^{2}+\sigma_{2}^{2}+\sigma_{3}^{2} .
$$

The Weyl tensor of $\mathbb{H H}^{1}$ vanishes, corresponding to the fact that it (and its boundary $\mathrm{S}^{3}$ ) are conformally flat.

The $\mu=k$ limit: The Bergman space Taking the $\mu=k$ limit of (3.7), we obtain

$$
g_{\mu \nu}^{(\mu=k, k)}: \mathrm{d} s^{2}=\frac{4}{\left(1-k^{2} r^{2}\right)^{2}}\left[\frac{\mathrm{d} r^{2}}{1+k^{2} r^{2}}+r^{2}\left(1+k^{2} r^{2}\right) \sigma_{3}^{2}\right]+\frac{4 r^{2}\left(\sigma_{1}^{2}+\sigma_{2}^{2}\right)}{1-k^{2} r^{2}} .
$$

This is the metric on the coset space $\widetilde{\mathbb{C P}^{2}}=\mathrm{SU}(2,1) / \mathrm{U}(2)$, albeit in a slightly nonstandard form. By performing the coordinate change

$$
r^{2}=\frac{\rho^{2}}{2-k^{2} \rho^{2}}
$$

we obtain the more usual Bergman (pseudo-Fubini-Study) metric for $\widetilde{\mathbb{C P}^{2}}$ :

$$
g_{\mu \nu}^{(k, k)}: \quad \mathrm{d} s^{2}=2 \frac{\mathrm{d} \rho^{2}+\rho^{2} \sigma_{3}^{2}}{\left(1-k^{2} \rho^{2}\right)^{2}}+2 \frac{\rho^{2}\left(\sigma_{1}^{2}+\sigma_{2}^{2}\right)}{\left(1-k^{2} \rho^{2}\right)} .
$$

We will thus loosely refer to the space $\widetilde{\mathbb{C P}^{2}}$ as the "Bergman space". The isometry group is now $\mathrm{SU}(2,1)$, and (according to [10]) becomes the conformal group of the boundary.

Note that the Bergman space is a Kähler manifold. So in this limit, our quaternionic Kähler metric actually becomes Kähler. As for the induced boundary metric, it is clear from (3.14) that as one approaches the boundary $(r \rightarrow 1 / k)$ the coefficient of $\sigma_{3}$ blows up faster that the others, and so the boundary metric degenerates ${ }^{9}$. Despite this degeneracy, the authors of [10] showed that one could obtain meaningful results for correlation functions in the boundary theory.

\section{Propagators for the known cases}

In trying to describe the boundary conformal field theory, a fundamental quantity is the bulkto-boundary propagator. This parametrises the coupling of a field in the bulk to its dual operator in the boundary theory, and is essential in calculating boundary correlation functions.

\footnotetext{
${ }^{9}$ Technically, since the metric is Kähler, what extends to the boundary is not a conformal structure, but a complex structure, and at the boundary we are left with a CR structure (which is generically a degenerate complex structure) [52.
} 
Although in this analysis we are interested only in scalar two-point functions, for which the bulk-to-boundary propagator is sufficient, we find it easier and more intuitive to deal with the bulk-to-bulk propagators, from which we can obtain the bulk-to-boundary ones via a limiting process.

We note the laplacian for the oblate Pedersen metric (3.1). (The corresponding laplacian for (3.7) can be found through $m=i \mu)$ :

$$
\begin{aligned}
\nabla^{2} & =\frac{1}{\sqrt{g}} \partial_{\mu} \sqrt{g} g^{\mu \nu} \partial_{\nu} \\
& =\frac{\left(1-k^{2} r^{2}\right)^{2}}{\left(1+m^{2} r^{2}\right)}\left[\frac{1}{4}\left(1+m^{2} k^{2} r^{4}\right) \partial_{r r}+\frac{1}{4 r\left(1-k^{2} r^{2}\right)}\left(3+k^{2} r^{2}+7 m^{2} k^{2} r^{4}-3 m^{2} k^{4} r^{6}\right) \partial_{r}\right. \\
& \left.+\frac{1}{r^{2}}\left\{\partial_{\theta \theta}+\cot \theta \partial_{\theta}+\csc ^{2} \theta\left[\partial_{\varphi \varphi}-2 \cos \theta \partial_{\varphi \psi}+\left(\sin ^{2} \theta \frac{\left(1+m^{2} r^{2}\right)^{2}}{1+m^{2} k^{2} r^{4}}+\cos ^{2} \theta\right) \partial_{\psi \psi}\right]\right\}\right]
\end{aligned}
$$

In general, a coordinate space Green's function $G\left(x_{r}, x_{s}\right)$ in four dimensions will depend on eight variables. Following [10], we use the $\mathrm{SU}(2) \times \mathrm{U}(1)$ isometry group to restrict propagators to depend on the following combinations of the angular parameters $\theta_{r}, \varphi_{r}, \psi_{r}, \theta_{s}, \varphi_{s}, \psi_{s}:{ }^{10}$

$$
U=\cos \frac{\theta_{r}}{2} \cos \frac{\theta_{s}}{2} e^{\frac{i}{2}\left(\psi_{s}-\psi_{r}+\varphi_{s}-\varphi_{r}\right)}+\sin \frac{\theta_{r}}{2} \sin \frac{\theta_{s}}{2} e^{\frac{i}{2}\left(\psi_{s}-\psi_{r}-\varphi_{s}+\varphi_{r}\right)} .
$$

and its conjugate

$$
\bar{U}=\cos \frac{\theta_{r}}{2} \cos \frac{\theta_{s}}{2} e^{-\frac{i}{2}\left(\psi_{s}-\psi_{r}+\varphi_{s}-\varphi_{r}\right)}+\sin \frac{\theta_{r}}{2} \sin \frac{\theta_{s}}{2} e^{-\frac{i}{2}\left(\psi_{s}-\psi_{r}-\varphi_{s}+\varphi_{r}\right)} .
$$

The Green's function should be symmetric in $U$ and $\bar{U}$, so we find it convenient to introduce the two combinations ${ }^{11}$ :

$$
\mathrm{v}_{1}:=U+\bar{U}=2 \cos \frac{\theta_{r}}{2} \cos \frac{\theta_{s}}{2} \cos \frac{1}{2}\left(\psi_{r}+\varphi_{r}-\psi_{s}-\varphi_{s}\right)+2 \sin \frac{\theta_{r}}{2} \sin \frac{\theta_{s}}{2} \cos \frac{1}{2}\left(\psi_{r}-\varphi_{r}-\psi_{s}+\varphi_{s}\right)
$$

and

$$
\mathrm{v}_{2}:=U \bar{U}=\frac{1}{2}\left\{1+\cos \theta_{r} \cos \theta_{s}+\sin \theta_{r} \sin \theta_{s} \cos \left(\varphi_{r}-\varphi_{s}\right)\right\} .
$$

So from now on we will treat the bulk propagators as functions of $r, s, \mathrm{v}_{1}, \mathrm{v}_{2}$. That is, we have used the isometries to reduce the number of variables from eight to four, and by doing so we have exhausted the available symmetries of the metric ${ }^{12}$. In these new variables, $x_{r}=x_{s}$ translates to $\left\{r=s, \mathrm{v}_{1}=2, \mathrm{v}_{2}=1\right\}$.

\footnotetext{
${ }^{10} \mathrm{In}[10, \mathrm{SU}(2) \times \mathrm{U}(1)$ appears as the isometry group of the boundary of the Bergman space, while the bulk isometry group is $\mathrm{SU}(2,1)$. In the more general QTN case both the bulk and the boundary have $\mathrm{SU}(2) \times \mathrm{U}(1)$ isometry group, and the same arguments apply.

${ }^{11} \mathrm{v}_{1} / 2$ is called $\mu$ in [36].

${ }^{12}$ Note that, unlike the definition of [10], our $U, \bar{U}$ are pure angular variables. This means that $\mathrm{v}_{1}, \mathrm{v}_{2}$ will always appear in Green's functions in the combinations $r s \mathrm{v}_{1}, r^{2} s^{2} \mathrm{v}_{2}$.
} 
It is now useful to rewrite the laplacian (4.1) in terms of $\mathrm{v}_{1}$ and $\mathrm{v}_{2}$ :

$$
\begin{aligned}
\nabla^{2}=\frac{\left(1-k^{2} r^{2}\right)^{2}}{\left(1+m^{2} r^{2}\right)}[ & \frac{1}{4}\left(1+m^{2} k^{2} r^{4}\right) \partial_{r r}+\frac{1}{4 r\left(1-k^{2} r^{2}\right)}\left(3+k^{2} r^{2}+7 m^{2} k^{2} r^{4}-3 m^{2} k^{4} r^{6}\right) \partial_{r} \\
& +\frac{1}{r^{2}}\left\{-1 / 4 \mathrm{v}_{1} \frac{\left(3+2 m^{2} r^{2}+2 m^{2} k^{2} r^{4}+m^{4} r^{4}\right)}{1+m^{2} k^{2} r^{4}} \partial_{\mathrm{v}_{1}}\right. \\
& +\frac{1+m^{2} k^{2} r^{4}-1 / 4 \mathrm{v}_{1}^{2}\left(1+m^{2} r^{2}\right)^{2}-m^{2} r^{2} \mathrm{v}_{2}\left(-2+k^{2} r^{2}-m^{2} r^{2}\right)}{1+m^{2} k^{2} r^{4}} \partial_{\mathrm{v}_{1} \mathrm{v}_{1}} \\
& \left.\left.+\left(1-\mathrm{v}_{2}\right) \mathrm{v}_{1} \partial_{\mathrm{v}_{1} \mathrm{v}_{2}}+\left(1-\mathrm{v}_{2}\right) \mathrm{v}_{2} \partial_{\mathrm{v}_{2} \mathrm{v}_{2}}-\left(2 \mathrm{v}_{2}-1\right) \partial_{\mathrm{v}_{2}}\right\}\right]
\end{aligned}
$$

In the following sections we give the known Green's functions for the various limiting cases of this laplacian (and the one related to it by $m=i \mu$ ). We use the notation $G^{(\mu, k)}$ or $G^{(m, k)}$ for the Green's functions corresponding to the Pedersen metrics (3.7) and (3.1) respectively. We will discuss two common possibilities for scalars propagating in a curved spacetime, conformal and minimal coupling. Note that since the scalar curvature of QTN is $\mathcal{R}=-12 k^{2}$, the conformally coupled laplacian is $\nabla^{2}-\frac{1}{6} \mathcal{R}=\nabla^{2}+2 k^{2}$.

\subsection{Green's Functions for $\mathbb{H H}^{1}$}

In this section we review a few well-known facts for propagators on euclidean $\mathrm{AdS}_{4}$. All this is completely standard material (54, 2, 55, 56, 57, 58, 59, , see also [39] for a review), and is only included for easy comparison with other limiting cases of QTN, and to familiarise the reader with our slightly non-standard notation. (A sketch of how one converts to the usual expressions for bulk-to-bulk and bulk-to-boundary propagators can be found in appendix B.)

One can easily invert the (massive) laplacian equation for $\mathbb{H H}^{1}$ by assuming that the Green's function is a function only of the chordal distance $u_{0}:=u^{(0, k)}$, where (e.g. [59]).

$$
u^{(0, k)}=\frac{2 k^{2}\left(r^{2}+s^{2}-r s \mathrm{v}_{1}\right)}{\left(1-k^{2} r^{2}\right)\left(1-k^{2} s^{2}\right)}
$$

So the chordal distance allows us to express the $\mu=0$ limit of the laplacian (4.6) in terms of only one variable:

$$
\nabla^{2}-M^{2}=2 k^{2}\left\{u_{0} \frac{u_{0}+2}{2} \partial_{u_{0} u_{0}}+2\left(u_{0}+1\right) \partial_{u_{0}}-\frac{M^{2}}{2 k^{2}}\right\}
$$

This is a hypergeometric equation whose solutions are known for arbitrary values of $M^{2}$. However, as mentioned, we shall concentrate on the following special cases:

Conformal coupling Here $M^{2}=-2 k^{2}$ and we obtain the two solutions

$$
\begin{aligned}
& G_{1}^{(0, k)}=C \cdot \frac{2 k^{2}}{u_{0}}=C \cdot \frac{\left(1-k^{2} r^{2}\right)\left(1-k^{2} s^{2}\right)}{r^{2}+s^{2}-r s \mathrm{v}_{1}} \\
& G_{2}^{(0, k)}=C \cdot \frac{4 k^{2}}{u_{0}\left(u_{0}+2\right)}=C \cdot \frac{\left(1-k^{2} r^{2}\right)^{2}\left(1-k^{2} s^{2}\right)^{2}}{\left(1+k^{4} r^{2} s^{2}-k^{2} r s \mathrm{v}_{1}\right)\left(r^{2}+s^{2}-r s \mathrm{v}_{1}\right)} .
\end{aligned}
$$


Notice that these propagators correspond to two different choices of boundary condition. This is the well known fact [60] that fields in $\mathrm{AdS}_{d+1}$ in the mass range $-d^{2} / 4<M^{2} / k^{2}<-d^{2} / 4+1$ can have two possible quantisations. We will return to this point in section 8 . Both propagators reduce to the massless flat space Green's function as we take the cosmological constant to zero $(k \rightarrow 0)$ :

$$
G_{1}^{(0, k)}, G_{2}^{(0, k)} \longrightarrow G^{(0,0)}=C \cdot \frac{1}{r^{2}+s^{2}-r s \mathrm{v}_{1}} .
$$

The normalisation factors $C$ are known for $\mathbb{H H}^{1}$, but since we are also interested in the more general QTN case we will not keep track of them. Instead, we simply normalise all propagators (in this and the following sections) so that they give the same flat-space Green's function as $k \rightarrow 0$.

From the bulk-to-bulk propagators one can find bulk-to-boundary propagators by taking the appropriate scaling limits $(61,62,)^{13}$ :

$$
K_{1}^{(0, k)}=\lim _{s \rightarrow 1 / k}\left\{\frac{G_{1}^{(0, k)}}{\left(1-k^{2} s^{2}\right)}\right\}=\frac{1-k^{2} r^{2}}{1+k^{2} r^{2}-k r \mathrm{v}_{1}}
$$

and

$$
K_{2}^{(0, k)}=\lim _{s \rightarrow 1 / k}\left\{\frac{G_{2}^{(0, k)}}{\left(1-k^{2} s^{2}\right)^{2}}\right\}=\left(\frac{1-k^{2} r^{2}}{1+k^{2} r^{2}-k r \mathrm{v}_{1}}\right)^{2} .
$$

We have normalised these and all subsequent bulk-to-boundary propagators to give 1 as $r \rightarrow 0$.

Minimal Coupling Here $M^{2}=0$ and we have only one solution (the other is a constant):

$$
G_{3}^{(0, k)}=C \cdot k^{2}\left[-\ln \left(1+\frac{2}{u_{0}}\right)+\frac{2}{u_{0}} \frac{u_{0}+1}{u_{0}+2}\right] .
$$

Notice that $G_{3}^{(0, k)}$ also reduces to the massless flat space Green's function when $k \rightarrow 0$, as it should. To obtain the bulk-to-boundary propagator, we need to take the limit

$$
K_{3}^{(0, k)}=\lim _{s \rightarrow 1 / k}\left\{\frac{G_{3}^{(0, k)}}{\left(1-k^{2} s^{2}\right)^{3}}\right\}=\left(\frac{1-k^{2} r^{2}}{1+k^{2} r^{2}-k r \mathrm{v}_{1}}\right)^{3} .
$$

\subsection{Green's Functions for $\widetilde{\mathbb{C P}^{2}}$}

In the case of the Bergman space, one can again simplify the laplacian equation considerably by assuming that the Green's function is a function only of the chordal distace, which we denote by $u_{1}:=u^{(\mu=k, k)}$. Expressions for the chordal distance and scalar Green's functions in the case of

\footnotetext{
${ }^{13}$ Of course, if we had wanted to be careful with normalisation factors, we would have had to first define a proper Dirichlet problem at $r=1 / k-\epsilon$ by analogy with [3, 63, 56, define the bulk-to-boundary propagators by normal derivatives of the bulk-to-bulk ones, and then take the regulator to zero. Or we could add appropriate boundary terms to the action as done e.g. in 62 . Then for $\operatorname{AdS}_{d+1}$ we would obtain the relative factor $1 /(2 \Delta-d)$ between $G$ and $K$ ( $\Delta$ is defined in section 8$)$.
} 
$\mathbb{C P}^{2}$ were found by Warner in 64. We simply extend these to the noncompact case by obvious changes in signs. After converting to our coordinate system for $\widetilde{\mathbb{C P}^{2}}$, we find

$$
u_{1}=2 k^{2} \frac{r^{2}+s^{2}+2 k^{2} r^{2} s^{2} \mathrm{v}_{2}-r s \sqrt{\left(1+k^{2} r^{2}\right)\left(1+k^{2} s^{2}\right)} \mathrm{v}_{1}}{\left(1-k^{2} r^{2}\right)\left(1-k^{2} s^{2}\right)} .
$$

With this ansatz the laplacian becomes

$$
\nabla^{2}-M^{2}=2 k^{2}\left\{u_{1}\left(u_{1}+1\right) \partial_{u_{1} u_{1}}+\left(2+3 u_{1}\right) \partial_{u_{1}}-\frac{M^{2}}{2 k^{2}}\right\}
$$

which is again a hypergeometric equation. As before we will restrict to the two special cases of conformally and minimally coupled scalars.

Conformal Coupling Setting $M^{2}=-2 k^{2}$ we again obtain two bulk-to-bulk propagators. The first one is easy to understand:

$$
G_{1}^{(k, k)}=C \cdot \frac{2 k^{2}}{u_{1}}=C \cdot \frac{\left(1-k^{2} r^{2}\right)\left(1-k^{2} s^{2}\right)}{r^{2}+s^{2}+2 k^{2} r^{2} s^{2} \mathrm{v}_{2}-r s \sqrt{\left(1+k^{2} r^{2}\right)\left(1+k^{2} s^{2}\right)} \mathrm{v}_{1}} .
$$

This Green's function has a nice boundary behaviour, vanishing as $\left(1-k^{2} r^{2}\right)^{1}$ at the boundary (where $r \rightarrow 1 / k$ ), like the corresponding mode in $\mathbb{H H}^{1}$. Taking the limit $k \rightarrow 0$, the Bergman space reduces to flat space and, as expected, we retrieve the flat space propagator as the $k \rightarrow 0$ limit of $G_{1}^{(k, k)}$.

From the bulk-to-bulk propagator we find the bulk-to-boundary propagator (which is essentially the Bergman kernel for the ball in $\mathbb{C}^{2}$ ) in the standard way

$$
K_{1}^{(k, k)}=\lim _{s \rightarrow 1 / k}\left\{\frac{G_{1}^{(k, k)}}{\left(1-k^{2} s^{2}\right)}\right\}=\frac{1-k^{2} r^{2}}{1+k^{2} r^{2}+2 k^{2} r^{2} \mathrm{v}_{2}-\sqrt{2} k r \sqrt{1+k^{2} r^{2}} \mathrm{v}_{1}}
$$

The second solution for conformal coupling is $\sim 1 / u_{1} \cdot \ln \left(1+u_{1}\right)$, i.e. at the boundary it behaves as $\left(1-k^{2} r^{2}\right) \ln \left(1-k^{2} r^{2}\right)$. This is very different from the corresponding mode in the $\mathbb{H H}^{1}$ case. As we will see at the end of section 8 , it might eventually become necessary to understand this mode better.

Minimal Coupling The solution for $M^{2}=0$ is

$$
G_{2}^{(k, k)}=C \cdot k^{2}\left[-\ln \left(1+\frac{1}{u_{1}}\right)+\frac{1}{u_{1}}\right]
$$

One can check that this also reduces to the flat space propagator as $k \rightarrow 0$. To obtain the bulk-to-boundary propagator, we need to take the limit

$$
K_{2}^{(k, k)}=\lim _{s \rightarrow 1 / k}\left\{\frac{G_{2}^{(k, k)}}{\left(1-k^{2} s^{2}\right)^{2}}\right\}=\left(\frac{1-k^{2} r^{2}}{1+k^{2} r^{2}+2 k^{2} r^{2} \mathrm{v}_{2}-\sqrt{2} k r \sqrt{1+k^{2} r^{2}} \mathrm{v}_{1}}\right)^{2} .
$$

This bulk-to-boundary propagator is the same one that appears in [10] for minimal coupling (to see this one has to convert to the metric (3.16) as explained in section 3). 


\subsection{Green's Function for euclidean Taub-NUT}

Since euclidean Taub-NUT (ETN) is Ricci-flat, there is no distinction between the conformally coupled and massless Green's function. The massless scalar Green's function for ETN has been found by Page [36]. In our parametrization, it is:

$$
G^{(m, 0)}=\frac{C}{2} \cdot \frac{2 \cosh \left(m^{2} \mathrm{w}\right)-\frac{\left(r^{2}+s^{2}\right)}{\mathrm{w}} \sinh \left(m^{2} \mathrm{w}\right)}{\left(r^{2}+s^{2}\right) \cosh \left(m^{2} \mathrm{w}\right)-2 \mathrm{w} \sinh \left(m^{2} \mathrm{w}\right)-r s \mathrm{v}_{1}},
$$

where

$$
\mathrm{w}:=\frac{1}{2} \sqrt{\left(r^{2}-s^{2}\right)^{2}-4 r^{2} s^{2}\left(\mathrm{v}_{2}-1\right)} .
$$

The euclidean Taub-NUT metric (3.5) reduces to flat space by taking $m \rightarrow 0$. Taking this limit of $G^{(m, 0)}$, we again obtain the flat-space Green's function $G^{(0,0)}$. ETN has zero cosmological constant, so we will not attempt to define bulk-to-boundary propagators since (for the moment) we are not interested in defining a boundary theory.

\section{The Twistor space of the Pedersen metric}

After reviewing the Green's functions for various special cases, we are now ready to consider the case of $\nabla^{2}-M^{2}$, where $\nabla^{2}$ is the full Pedersen laplacian (4.6). The calculation we will perform is similar in spirit to the one by Page in [36], where the Green's functions for scalar fields on multi-Taub-NUT manifolds and ALE multi-instantons were produced.

As in 36, the objective is to find functions of the coordinates that vanish when two points are null separated. In 36] these functions were found using Hamilton-Jacobi methods. We prefer to use twistor methods in order to connect with the treatment of [25]. This discussion is rather long and the reader who is not interested in twistors may prefer to skip to (6.31).

\subsection{More on the Pedersen metric}

In preparation for the description of the twistor space, we briefly review the main steps of the construction of the metric on QTN by Pedersen in [25]. Essentially the construction of [25] is similar to that of Gibbons and Hawking [65] for the hyperkähler case: One considers a $\mathrm{U}(1)$ monopole over a 3 -dimensional riemannian manifold $X^{3}$. This $\mathrm{U}(1)$ monopole is just a connection $A$ (the "gauge potential") on a principal U(1) bundle fibring over $X^{3}$ along with a function $V$ (the "Higgs field") that is related to the curvature of $A$ by the Bogomolny equation

$$
\mathrm{d} A=-\star \mathrm{d} V
$$

With this choice of sign the conformal structure

$$
V \mathrm{~d} s_{X^{3}}^{2}+V^{-1}(\mathrm{~d} \tau+A)^{2}
$$

(where $\mathrm{d} s_{X^{3}}^{2}$ is the metric on $X^{3}$ ) is anti-self-dual when $X^{3}$ has constant curvature. To obtain an Einstein space from this conformal structure, one has to multiply by a suitable function $F(\chi)$. So given a choice for $V$ satisfying (5.1) on $X^{3}$, and a corresponding choice for $F(\chi)$, the metric

$$
\mathrm{d} s^{2}=F^{2}(\chi)\left(V \mathrm{~d} s_{X^{3}}^{2}+V^{-1}(\mathrm{~d} \tau+A)^{2}\right)
$$


will be Einstein. The flat case $X^{3}=\mathbb{R}^{3}$ is the Gibbons-Hawking ansatz, which leads to a zero scalar curvature $4-$ manifold (then $F(\chi)=1$ ). Pedersen's ansatz replaces $X^{3}$ by $\mathrm{S}^{3}$ and the 3 -metric is chosen to be $\mathrm{d} s_{\mathrm{S}^{3}}^{2}=\mathrm{d} \chi^{2}+4 \sin ^{2} \chi\left(\sigma_{1}^{2}+\sigma_{2}^{2}\right)$. A choice that satisfies (5.1) is $(V, A)=(1+k / m \cot \chi,-k / m \cos \theta \mathrm{d} \varphi)$ and after multiplying by a suitable conformal factor (and rescaling $\tau=-2 k / m \cdot \psi$ ) one obtains an anti-self-dual Einstein 4-metric with negative cosmological constant [25]:

$$
k^{2} \mathrm{~d} s^{2}=\frac{1}{\left(\cos \chi-\frac{k}{m} \sin \chi\right)^{2}}\left[\left(1+\frac{k}{m} \cot \chi\right)\left\{\mathrm{d} \chi^{2}+4 \sin ^{2} \chi\left(\sigma_{1}^{2}+\sigma_{2}^{2}\right)\right\}+\frac{4 k^{2} \sigma_{3}^{2}}{m^{2}\left(1+\frac{k}{m} \cot \chi\right)}\right] .
$$

This is the "trigonometric" case in [52]. The coordinate change

$$
\cos \chi=\frac{1}{\sqrt{1+m^{2} k^{2} r^{4}}}
$$

shows that it is equivalent to the original "oblate" Pedersen metric (3.1).

There is another possibility that gives a negative cosmological constant, which is to consider $X^{3}=H^{3}$, the three dimensional hyperbolic space, with metric $\mathrm{d} s_{H^{3}}^{2}=\mathrm{d} \chi^{2}+4 \sinh ^{2} \chi\left(\sigma_{1}^{2}+\sigma_{2}^{2}\right)$. Then the Einstein 4-metric that arises is [52]:

$$
k^{2} \mathrm{~d} s^{2}=\frac{-1}{\left(\cosh \chi-\frac{k}{\mu} \sinh \chi\right)^{2}}\left[\left(1-\frac{k}{\mu} \operatorname{coth} \chi\right)\left\{\mathrm{d} \chi^{2}+4 \sinh ^{2} \chi\left(\sigma_{1}^{2}+\sigma_{2}^{2}\right)\right\}+\frac{4 k^{2} / \mu^{2} \sigma_{3}^{2}}{\left(1-\frac{k}{\mu} \operatorname{coth} \chi\right)}\right] .
$$

In this "hyperbolic" case we can perform the coordinate transformation

$$
\cosh \chi=\frac{1}{\sqrt{1-\mu^{2} k^{2} r^{4}}}
$$

to see that it is the same as the prolate Pedersen metric (3.7). Thus the analytic continuation (3.6) simply takes us from the trigonometric case to the hyperbolic one.

\subsection{The twistor space}

In the previous section we saw how to obtain an Einstein space with anti-self-dual conformal structure via a $\mathrm{U}(1)$ monopole over a three-dimensional space of constant curvature. There is a beautiful description of this situation in terms of twistor theory [66, 67. Although all the material in this section can be found (in much greater detail!) in the literature, in the interest of completeness we will attempt to review the path leading to the twistor space of our metric. Much of this and the following subsection is taken verbatim from [25] ${ }^{14}$. First we need to give a few definitions.

The twistor space of a self-dual ${ }^{15}$ four-dimensional Riemannian manifold $M$ is the projective bundle $Z=P\left(V_{-}\right)$of anti-self-dual spinors, and is a complex three-manifold 66, 69, 70, 71]. The crucial property of $Z$ is that its complex structure encodes the conformal structure of $M$.

\footnotetext{
${ }^{14}$ We apologise in advance to any mathematician readers for what is certainly a naive and oversimplified exposition of this elegant but often delicate construction.

${ }^{15}$ By self-dual we mean that the Weyl tensor is self-dual. We switched to self-dual language for consistency with the twistor literature, but the following discussion can be adapted to the anti-self-dual case simply by replacing "self-dual" with "anti-self-dual" everywhere.
} 
The way this works is, roughly, the following: There is a holomorphic projection $Z \rightarrow \mathbb{C P}^{1}$, whose sections are called twistor lines. These lines (which are rational curves-copies of $\mathbb{C P}^{1}-$ that have normal bundle $\mathcal{O}(1) \oplus \mathcal{O}(1)$ ) belong to a four complex-dimensional family, and their parameter space defines a complex self-dual conformal structure. Imposing reality under the real structure of $Z$ reduces this to the real self-dual conformal structure of $M$. (This construction easily generalises to hyperkähler and quaternionic Kähler manifolds in higher dimensions.)

LeBrun [26] showed the following fact, which underlies Pedersen's construction: If $N_{C}$ is a three-complex dimensional manifold with holomorphic metric (with an added property of being geodesically convex), then the space of unparametrised null geodesics of $N_{C}$ is the twistor space $Z$ of a four-complex-dimensional manifold $M_{C}$, which satisfies the self-dual Einstein equations with negative cosmological constant. The 3 -manifold $N_{C}$ appears as the conformal infinity (in the sense of Penrose) of the 4-manifold $M_{C}$. Now if $N_{C}$ is the complexification of a real-analytic 3-manifold $N$, using the real structure of $Z$ we can find a real slice $M$ of $M_{C}$. In this way we can describe how a three (real) dimensional manifold becomes the conformal infinity of a four (real) dimensional self-dual Einstein space with negative cosmological constant.

We now see that to apply the LeBrun construction to the Berger sphere (1.2) in order to find the twistor space $Z$ of the filling-in manifold $M$, Pedersen had to somehow describe the space of unparametrised null geodesics of the Berger sphere. By using an analogy with the motion of a free rigid body around a fixed point, he was able to show that $Z$ can be described as a line bundle over plane sections of the quadric in $\mathbb{C P}^{3}$. Using the notation $z_{i}$ for homogeneous coordinates on $\mathbb{C P}^{3}$, this quadric is given by

$$
Q: z_{1}^{2}+z_{2}^{2}+z_{3}^{2}+\nu^{2} z_{4}^{2}=0 \text {. }
$$

The parameter $\nu$ is related to the squashing parameter $\lambda$ in (1.2) by $\nu^{2}=\lambda(1-\lambda)$. By plane sections we mean the conics obtained by intersections of the quadric with certain planes in $\mathbb{C P}^{3}$. We will not go deeper into understanding the space of null geodesics, because there is an alternate description of $Z$ that will prove more useful.

To provide that description we need to introduce the concept of the minitwistor space of an Einstein-Weyl 3-manifold $X[72,67]^{16}$. This is a complex two-manifold that contains certain "special" rational curves (again called twistor lines) with normal bundle $\mathcal{O}(2)$ which can be shown to form a three-parameter family. So the Einstein-Weyl manifold $X$ appears as the parameter space of these special curves. There is a way [67] to pass from the twistor space $Z$ of a four-dimensional self-dual manifold $M$ to the minitwistor space of a three-dimensional Einstein-Weyl manifold $X$ that appears as the space of trajectories of a conformal motion on $M$, by suitably factoring $Z$ with a holomorphic vector field. This may sound vague but in the cases we are interested in, the relation between twistor and minitwistor spaces becomes very precise [25, 52]:

The twistor space $Z$ of a $\mathrm{U}(1)$ monopole over a space $X^{3}$ of constant curvature is given by a holomorphic $\mathbb{C}^{*}$-bundle over the minitwistor space of $X^{3}$, of degree zero on each twistor line of the minitwistor space.

To see the relevance of this, recall that in the previous section we showed how the Pedersen metric is constructed as a $\mathrm{U}(1)$ monopole over $\mathrm{S}^{3}$ or $H^{3}$. So we now have a way to construct

\footnotetext{
${ }^{16}$ An Einstein-Weyl manifold with a conformal metric $g_{i j}$ satisfies $\mathcal{R}_{(i j)}=\Lambda g_{i j}$, where $\mathcal{R}_{i j}$ is the (possibly nonsymmetric) Ricci tensor of a generically non-Levi-Civita connection [67. We will only consider the special case of Einstein's equations, which in three dimensions imply constant curvature.
} 
the twistor space of the Pedersen metric. We will restrict to the oblate case for which $X^{3}$ is simply round $\mathrm{S}^{3}$. The minitwistor space of $\mathrm{S}^{3}$ turns out to be just the quadric in $\mathbb{C P}^{3}$. This is of course not a coincidence since there is a close connection between the monopole and the LeBrun descriptions.

The quadric, then, plays a dual role: It parametrises the null geodesics of the squashed threesphere and also encodes, through its twistor lines, a round three-sphere $\mathrm{S}^{3}$. In the following we will use this second description to construct $Z$ and then, at the end, revert to the first one (since we are interested in the conformal infinity of QTN and not in its description as a U(1) monopole). The fact that the quadric (5.8) somehow encodes a round sphere will become clearer if we introduce affine coordinates. To do that, we need to set up patches. First, define the lines

$$
\begin{aligned}
l_{1}: z_{2}+i z_{3}=0 & \wedge z_{1}+i \nu z_{4}=0, \\
l_{2}: z_{2}+i z_{3}=0 & \wedge z_{1}-i \nu z_{4}=0, \\
m_{1}: z_{2}-i z_{3}=0 & \wedge z_{1}+i \nu z_{4}=0 \\
m_{2}: z_{2}-i z_{3}=0 & \wedge z_{1}-i \nu z_{4}=0 .
\end{aligned}
$$

On the patch $U_{1}=Q \backslash\left(l_{1} \cup l_{2}\right)$ we will use the following coordinates:

$$
\zeta=\frac{z_{1}+i \nu z_{4}}{z_{2}+i z_{3}}, \quad \eta=\frac{z_{1}-i \nu z_{4}}{z_{2}+i z_{3}}
$$

while on the other patch $U_{2}=Q \backslash\left(m_{1} \cup m_{2}\right)$ a valid coordinate system is

$$
\tilde{\zeta}=\frac{z_{1}-i \nu z_{4}}{z_{2}-i z_{3}}, \quad \tilde{\eta}=\frac{z_{1}+i \nu z_{4}}{z_{2}-i z_{3}}
$$

Notice that the coordinates on the overlap of the two patches are related simply by $\tilde{\zeta}=$ $-1 / \zeta, \tilde{\eta}=-1 / \eta$. Thus with this choice of affine coordinates we have exhibited the quadric $Q$ as the product $\mathbb{C P}^{1} \times \mathbb{C P}^{1}$. As stated above, the fact that $Q$ is the minitwistor space of $\mathrm{S}^{3}$ means that we can find special curves (the twistor lines) in $Q$ whose parameter space is $\mathrm{S}^{3}$. These twistor lines turn out to be plane sections, i.e. intersections of the quadric with hyperplanes in $\mathbb{C P}^{3}$. As explained in [25] in affine coordinates this translates to the condition

$$
\eta=\frac{-\bar{b}+\bar{a} \zeta}{a+b \zeta}
$$

We have introduced the complex parameters $a, b$ that satisfy $a \bar{a}+b \bar{b}=1$, i.e. they are coordinates on a round $\mathrm{S}^{3}$. This is how $\mathrm{S}^{3}$ appears as the parameter space of the twistor lines of its corresponding minitwistor space $Q$.

Having found the twistor lines of the quadric, the next step (according to the general description above) is to construct a line bundle $L$, trivial over these twistor lines. This bundle will generate both the conformal structure we aim for, and a $U(1)$ monopole described by $(V, A)$ above. Assume we have such a line bundle $L$, defined on a neighbourhood of a plane section of the quadric. The fact that $L$ is trivial over plane sections means that if a section of $L$ is given by the holomorphic functions $\left(\sigma_{1}, \sigma_{2}\right)$ on the coordinate patches $U_{1}, U_{2}$ defined above, and further if $\psi_{12}$ is the transition function with respect to $U_{1}, U_{2}$, then (on the plane sections)

$$
\sigma_{1}=\psi_{12} \sigma_{2}
$$


Since we aim for a Higgs field $V=1+k / m \cot \chi$, Pedersen's idea is to construct $L$ as the direct product of (suitable powers of) the line bundles $P$ and $T$ that give $V=i \cot \chi$ and $V=1$, respectively. The bundle $P$ is shown to have transition function

$$
\psi_{12}^{(P)}(\zeta, \eta)=\frac{(\zeta-\eta)^{2}}{\zeta \eta}
$$

while the transition function for $T$ is just

$$
\psi_{12}^{(T)}(\zeta, \eta)=\frac{\eta}{\zeta}
$$

To introduce the parameter $m / k$ we can consider powers of the bundle $T$. So in the end the transition function of the tensor product $L=T^{i \frac{m}{k}} \otimes P$ is simply

$$
\psi_{12}=\left(\psi_{12}^{(T)}\right)^{i \frac{m}{k}} \cdot \psi_{12}^{(P)} .
$$

We have thus constructed a line bundle, trivial over plane sections of the quadric, that naively gives the required monopole behaviour. Remarkably, Pedersen actually proves that this simple guess is correct, and thus (using the correspondence between the monopole description and the LeBrun construction) that

The twistor space $Z$ of the Pedersen metric in the $\mathrm{S}^{3}$ (trigonometric) case (i.e. the case that gives a boundary conformal structure $\sigma_{1}^{2}+\sigma_{2}^{2}+\lambda \sigma_{3}^{2}$ with $\lambda<1$ ) is the line bundle (minus the zero section)

$$
L:=\left(T^{i \frac{m}{k}} \otimes P\right) \backslash 0
$$

defined on a neighbourhood of a plane section of the quadric $Q$ in $\mathbb{C P}^{3}$.

\subsection{The real twistor lines}

In the previous section we obtained a description of the twistor space $Z$ of the Pedersen metric in terms of a line bundle over plane sections of the quadric. We want to better understand the null structure of the Pedersen metric, so we need to examine the twistor lines of $Z$.

First notice that the real structure on the quadric given, in terms of the homogeneous coordinates, by $\tau:\left(z_{1}, \ldots, z_{4}\right) \rightarrow\left(\bar{z}_{1}, \ldots, \bar{z}_{4}\right)$ takes the following form with respect to our affine coordinates $(\zeta, \eta)$ :

$$
\tau:(\zeta, \eta) \rightarrow(-1 / \bar{\zeta},-1 / \bar{\eta})
$$

It is easy to see that the plane sections given by (5.12) above are real with respect to this real structure. As we saw in the previous section, these real plane sections parametrise an $S^{3}$ via the coordinates $a, \bar{a}, b, \bar{b}$. The twistor lines of $Z$ should have one extra parameter, the fibre coordinate $\tau$ that describes the real sections of $L$ over the real plane sections ${ }^{17}$. The reason that we restrict to real sections is that we want $\tau$ to be real, to give a total of four real coordinates on QTN.

To find the real sections of $L$, we need to extend the real structure (5.18) on the quadric to the full twistor space. This can be done in various ways. Since $L$ doesn't contain the zero

\footnotetext{
${ }^{17}$ We expect no confusion to arise between the real structure and the fibre coordinate, both denoted by the letter $\tau$. We note also in passing that this is not the same $\tau$ as the one in (5.2).
} 
section, we can choose the following real structure on the fibre: $\tau: z \rightarrow \pm 1 / \bar{z}$. Then the full real structure on $L$ is

$$
\tau:(\zeta, \eta, \sigma) \rightarrow\left(-1 / \bar{\zeta},-1 / \bar{\eta}, \pm \bar{\psi}_{12}(\zeta, \eta) / \bar{\sigma}\right)
$$

Here $\psi_{12}$ is the transition function defined in (5.16). We thus have all the necessary ingredients to describe the real section $\left(\sigma_{1}, \sigma_{2}\right)$ on the real plane sections. First, substituting (5.12) in (5.13) (with $\psi_{12}$ from (5.16) ) we have [25]

$$
\psi_{12}=\left(\frac{-\bar{b}+\bar{a} \zeta}{\zeta(a+b \zeta)}\right)^{i \frac{m}{k}} \cdot\left(\frac{b^{2}(\zeta-\alpha)^{2}(\zeta-\beta)^{2}}{\zeta(a+b \zeta)(-\bar{b}+\bar{a} \zeta)}\right)=\sigma_{1} \cdot \sigma_{2}^{-1} .
$$

Here we have introduced the roots $\alpha$ and $\beta$ of the equation $b \zeta^{2}+(a-\bar{a}) \zeta+\bar{b}=0$. We choose

$$
\alpha=\frac{\bar{a}-a+\sqrt{d}}{2 b}, \quad \beta=\frac{\bar{a}-a-\sqrt{d}}{2 b},
$$

with $d=(a-\bar{a})^{2}-4 b \bar{b}$ the discriminant. Second, the requirement of reality under (5.19), can be written as

$$
\sigma_{1}(\tau(\zeta))= \pm 1 / \bar{\sigma}_{2}(\zeta)
$$

A choice for $\sigma_{1}, \sigma_{2}$ that satisfies both (5.20) and (5.22) is

$$
\left(\sigma_{1}, \sigma_{2}\right)=\left(i e^{i \tau} \frac{b}{\beta} \frac{(\zeta-\beta)^{2}}{(a+b \zeta)^{i m / k+1}}, \frac{i e^{i \tau}}{b \beta} \frac{\zeta^{i m / k+1}}{(\zeta-\alpha)^{2}(\bar{a} \zeta-\bar{b})^{i m / k-1}}\right) .
$$

We finally have the full description of the real twistor lines over the real plane sections: They are given by (restricting to the patch $U_{1}$ from now on):

$$
\eta=\frac{-\bar{b}+\bar{a} \zeta}{a+b \zeta}
$$

and

$$
\sigma=i e^{i \tau} \frac{b}{\beta} \frac{(\zeta-\beta)^{2}}{(a+b \zeta)^{i m / k+1}} .
$$

In the next section we will finally start building on Pedersen's work by using these twistor lines to construct the functions of null separation of the Pedersen metric.

\section{The functions of null separation}

Having found the twistor lines, we can now use Penrose's nonlinear graviton construction 68 . 69, 70, 71 to derive the functions of null separation. We use the following well-known fact:Two points $\left(\right.$ say $\left.x_{r}, x_{s}\right)$ on the 4 -dimensional manifold parametrised by $a, b, \bar{a}, \bar{b}, \tau$ are null separated if their corresponding twistor lines intersect. So the condition for $x_{r}$ and $x_{s}$ to be null separated is that the two equations

$$
\text { (I): } \eta_{r} \doteq \eta_{s}, \quad(\mathrm{II}): \quad \sigma_{r} \doteq \sigma_{s}
$$

have a common root. Here $\eta_{r}, \eta_{s}, \sigma_{r}, \sigma_{s}$ are the twistor lines evaluated at the points $x_{r}$ and $x_{s}$, with corresponding coordinates $a_{r}, b_{r}, \bar{a}_{r}, \bar{b}_{r}, \tau_{r}$ and $a_{s}, b_{s}, \bar{a}_{s}, \bar{b}_{s}, \tau_{s}$. (We will use the symbol $\doteq$ 
to denote equality on the null cone.) Clearly this description applies to the whole null cone, i.e. for arbitrary separation of the two points $x_{r}$ and $x_{s}$. Often (as in 70 for instance) one considers the infinitesimal version of (6.1) (i.e. $x_{r}$ and $x_{s}$ close together), which is all that is required to obtain the conformal structure (and eventually the Einstein manifold) described by $Z$. Here we are looking for functions that vanish on the whole null cone, so we need to keep $x_{r}$ and $x_{s}$ at arbitrary separation.

The coordinates $a, \bar{a}, b, \bar{b}, \tau$ are the ones adapted to the description of QTN as a U(1) bundle over $\mathrm{S}^{3}$ with the standard round metric. As discussed earlier, we need to convert these to the coordinates $r, \theta, \varphi, \psi$ that are more relevant to the description of QTN as the manifold giving the Berger sphere as its conformal infinity.

The transformations that achieve this are [25]:

$$
a=\frac{1+i m k r^{2} \cos \theta}{\sqrt{1+m^{2} k^{2} r^{4}}} \quad, \quad b=\frac{m k r^{2} \sin \theta}{\sqrt{1+m^{2} k^{2} r^{4}}} e^{i \varphi}
$$

and $^{18}$

$$
\tau=-\psi-\varphi+\arctan m k r^{2}
$$

Note also that the expression $\beta$ that appears in $\sigma$ is now

$$
\beta=-i \frac{(1+\cos \theta)}{\sin \theta} e^{-i \varphi}
$$

So we now begin the rather arduous process of converting the conditions (I) and (II) to expressions involving the points $x_{r}=\left(r, \theta_{r}, \varphi_{r}, \psi_{r}\right)$ and $x_{s}=\left(s, \theta_{s}, \varphi_{s}, \psi_{s}\right)$. The first condition (I) is

$$
\frac{-\bar{b}_{r}+\bar{a}_{r} \zeta}{a_{r}+b_{r} \zeta} \doteq \frac{-\bar{b}_{s}+\bar{a}_{s} \zeta}{a_{s}+b_{s} \zeta}
$$

which has two solutions:

$$
\zeta_{ \pm}=\frac{1}{2} \frac{\bar{b}_{r} b_{s}+\bar{a}_{s} a_{r}-\bar{b}_{s} b_{r}-\bar{a}_{r} a_{s} \pm \sqrt{\Delta_{0}}}{\bar{a}_{r} b_{s}-\bar{a}_{s} b_{r}}
$$

with the discriminant $\Delta_{0}$ given by

$$
\begin{aligned}
\Delta_{0}= & \bar{b}_{r}^{2} b_{s}^{2}+\bar{a}_{r}^{2} a_{s}^{2}+\bar{b}_{s}^{2} b_{r}^{2}+\bar{a}_{s}^{2} a_{r}^{2}-4\left(\bar{a}_{r} b_{s} \bar{b}_{s} a_{r}+\bar{a}_{s} b_{r} \bar{b}_{r} a_{s}\right) \\
& +2\left(\bar{b}_{r} b_{s} \bar{a}_{r} a_{s}-\bar{b}_{r} b_{s} \bar{b}_{s} b_{r}+\bar{b}_{r} b_{s} \bar{a}_{s} a_{r}+\bar{a}_{r} a_{s} \bar{b}_{s} b_{r}-\bar{a}_{r} a_{s} \bar{a}_{s} a_{r}+\bar{b}_{s} b_{r} \bar{a}_{s} a_{r}\right) .
\end{aligned}
$$

Using the explicit expressions

$$
\begin{aligned}
a_{r} & =\frac{1+i m k r^{2} \cos \theta_{r}}{\sqrt{1+m^{2} k^{2} r^{4}}}, & a_{s} & =\frac{1+i m k s^{2} \cos \theta_{s}}{\sqrt{1+m^{2} k^{2} s^{4}}}, \\
b_{r} & =\frac{m k r^{2} \sin \theta_{r} e^{i \varphi_{r}}}{\sqrt{1+m^{2} k^{2} r^{4}}} \quad, & b_{s} & =\frac{m k s^{2} \sin \theta_{s} e^{i \varphi_{s}}}{\sqrt{1+m^{2} k^{2} s^{4}}},
\end{aligned}
$$

\footnotetext{
${ }^{18}$ Our variable $\psi$ differs by a sign from the one used by Pedersen. This is required to match the conventions for the $\mathrm{SU}(2)$ one-forms: As mentioned earlier, in [25] $\mathrm{d} \sigma_{i}=\sum \epsilon_{i j k} \sigma_{j} \wedge \sigma_{k}$, while we use $\mathrm{d} \sigma_{i}=-\sum \epsilon_{i j k} \sigma_{j} \wedge \sigma_{k}$. Combined with a different choice for $\omega$ in [25], p. 51 (namely, $\omega=-\cos \theta \mathrm{d} \varphi+\mathrm{d}(\varphi-\chi)$ ), that is required by our anti-self-dual ansatz (5.1), $\psi \rightarrow-\psi$ takes $\sigma_{3} \rightarrow-\sigma_{3}$.
} 
and the definition of $\mathrm{v}_{2}$ from (4.5), we rewrite $\Delta_{0}$ as

$$
\Delta_{0}=-\frac{4 m^{2} k^{2}}{\left(1+m^{2} k^{2} r^{4}\right)\left(1+m^{2} k^{2} s^{4}\right)} \Delta
$$

where

$$
\Delta=\left(r^{2}-s^{2}\right)^{2}-4 r^{2} s^{2}\left(\mathrm{v}_{2}-1\right)-4 m^{2} k^{2} r^{4} s^{4} \mathrm{v}_{2}\left(\mathrm{v}_{2}-1\right) .
$$

So the final expression for $\zeta_{+}$and $\zeta_{-}$is

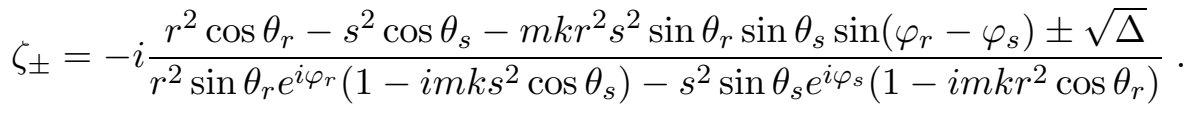

It is clear that $\zeta_{+} \leftrightarrow \zeta_{-}$if we interchange $x_{r}$ with $x_{s}$. Also, it can be checked that $\bar{\zeta}_{+} \zeta_{-}=-1$.

Since condition (I) has two solutions, substituting into (II) we obtain two conditions for the sigmas:

$$
\sigma_{r}\left(\zeta_{+}\right) \doteq \sigma_{s}\left(\zeta_{+}\right) \text {and } \sigma_{r}\left(\zeta_{-}\right) \doteq \sigma_{s}\left(\zeta_{-}\right)
$$

So we are led to consider the following two functions that are equal to unity when the points $x_{r}$ and $x_{s}$ are null separated:

$$
\mathcal{A}_{+}=\frac{\sigma_{r}\left(\zeta_{+}\right)}{\sigma_{s}\left(\zeta_{+}\right)} \doteq 1 \text { and } \mathcal{A}_{-}=\frac{\sigma_{r}\left(\zeta_{-}\right)}{\sigma_{s}\left(\zeta_{-}\right)} \doteq 1
$$

To construct Green's functions it is useful to combine $\mathcal{A}_{+}$and $\mathcal{A}_{-}$so as to obtain expressions that have definite symmetry properties under $x_{r} \leftrightarrow x_{s}$. To do that we notice first of all that under $x_{r} \leftrightarrow x_{s}$, we have

$$
\mathcal{A}_{+} \leftrightarrow \frac{1}{\mathcal{A}_{-}}
$$

(To see this, consider that under $x_{r} \leftrightarrow x_{s}$ we clearly have $a_{r} \leftrightarrow a_{s}, b_{r} \leftrightarrow b_{s}$ and $\beta_{r} \leftrightarrow \beta_{s}$ and as mentioned also $\zeta_{+} \leftrightarrow \zeta_{-}$. Then $x_{r} \leftrightarrow x_{s}$ takes $\sigma_{r}\left(\zeta_{+}\right) \leftrightarrow \sigma_{s}\left(\zeta_{-}\right)$and $\sigma_{r}\left(\zeta_{-}\right) \leftrightarrow \sigma_{s}\left(\zeta_{+}\right)$.)

We thus define a symmetric (under $x_{r} \leftrightarrow x_{s}$ ) function $\mathcal{U}\left(x_{r}, x_{s}\right)$ and an antisymmetric function $\mathcal{T}\left(x_{r}, x_{s}\right)$ by

$$
e^{-4 \mathcal{U}\left(x_{r}, x_{s}\right)}=\frac{\mathcal{A}_{+}}{\mathcal{A}_{-}} \text {and } e^{-4 i \mathcal{T}\left(x_{r}, x_{s}\right)}=\mathcal{A}_{+} \mathcal{A}_{-}
$$

We choose the coefficients of $\mathcal{U}$ and $\mathcal{T}$ for consistency with Page 36. We will see that these two functions reduce to the ones found in that article in the limit of zero cosmological constant.

Now we have to calculate the functions $\mathcal{U}\left(x_{r}, x_{s}\right)$ and $\mathcal{T}\left(x_{r}, x_{s}\right)$. We start by finding $\mathcal{A}_{+} / \mathcal{A}_{-}$:

$$
\frac{\mathcal{A}_{+}}{\mathcal{A}_{-}}=\frac{\sigma_{r}\left(\zeta_{+}\right)}{\sigma_{s}\left(\zeta_{+}\right)} \frac{\sigma_{s}\left(\zeta_{-}\right)}{\sigma_{r}\left(\zeta_{-}\right)}=\left\{\frac{\left(\zeta_{+}-\beta_{r}\right)\left(\zeta_{-}-\beta_{s}\right)}{\left(\zeta_{+}-\beta_{s}\right)\left(\zeta_{-}-\beta_{r}\right)}\right\}^{2}\left\{\frac{\left(a_{s}+b_{s} \zeta_{+}\right)\left(a_{r}+b_{r} \zeta_{-}\right)}{\left(a_{r}+b_{r} \zeta_{+}\right)\left(a_{s}+b_{s} \zeta_{-}\right)}\right\}^{i \frac{m}{k}+1}
$$

A rather long calculation gives the simple answer

$$
\frac{\left(\zeta_{+}-\beta_{r}\right)\left(\zeta_{-}-\beta_{s}\right)}{\left(\zeta_{+}-\beta_{s}\right)\left(\zeta_{-}-\beta_{r}\right)}=\frac{r^{2}+s^{2}-\sqrt{\Delta}-2 i m k r^{2} s^{2} \mathrm{v}_{2}}{r^{2}+s^{2}+\sqrt{\Delta}-2 i m k r^{2} s^{2} \mathrm{v}_{2}}
$$


and also

$$
\frac{\left(a_{s}+b_{s} \zeta_{+}\right)\left(a_{r}+b_{r} \zeta_{-}\right)}{\left(a_{r}+b_{r} \zeta_{+}\right)\left(a_{s}+b_{s} \zeta_{-}\right)}=\frac{1+i m k \sqrt{\Delta}+m^{2} k^{2} r^{2} s^{2}\left(2 \mathrm{v}_{2}-1\right)}{1-i m k \sqrt{\Delta}+m^{2} k^{2} r^{2} s^{2}\left(2 \mathrm{v}_{2}-1\right)} .
$$

Combining these expressions, we obtain the final result for the symmetric combination

$$
\frac{\mathcal{A}_{+}}{\mathcal{A}_{-}}=\left\{\frac{r^{2}+s^{2}-\sqrt{\Delta}-2 i m k r^{2} s^{2} \mathrm{v}_{2}}{r^{2}+s^{2}+\sqrt{\Delta}-2 i m k r^{2} s^{2} \mathrm{v}_{2}}\right\}^{2}\left\{\frac{1+i m k \sqrt{\Delta}+m^{2} k^{2} r^{2} s^{2}\left(2 \mathrm{v}_{2}-1\right)}{1-i m k \sqrt{\Delta}+m^{2} k^{2} r^{2} s^{2}\left(2 \mathrm{v}_{2}-1\right)}\right\}^{1+i \frac{m}{k}} .
$$

Now we turn to the antisymmetric combination $\mathcal{A}_{+} \mathcal{A}_{-}$. We have:

$$
\mathcal{A}_{+} \mathcal{A}_{-}=\frac{\sigma_{r}\left(\zeta_{+}\right)}{\sigma_{s}\left(\zeta_{+}\right)} \frac{\sigma_{r}\left(\zeta_{-}\right)}{\sigma_{s}\left(\zeta_{-}\right)}=\frac{e^{2 i \tau_{r}}}{e^{2 i \tau_{s}}} \frac{b_{r}^{2}}{b_{s}^{2}} \frac{\beta_{s}^{2}}{\beta_{r}^{2}}\left\{\frac{\left(\zeta_{+}-\beta_{r}\right)\left(\zeta_{-}-\beta_{r}\right)}{\left(\zeta_{+}-\beta_{s}\right)\left(\zeta_{-}-\beta_{s}\right)}\right\}^{2}\left\{\frac{\left(a_{s}+b_{s} \zeta_{+}\right)\left(a_{s}+b_{s} \zeta_{-}\right)}{\left(a_{r}+b_{r} \zeta_{+}\right)\left(a_{r}+b_{r} \zeta_{-}\right)}\right\}^{1+\frac{i m}{k}} .
$$

We will perform this calculation in steps. First we easily derive

$$
\frac{b_{r}^{2}}{b_{s}^{2}}=\frac{\left(1+m^{2} k^{2} s^{4}\right) r^{4} \sin ^{2} \theta_{r}}{\left(1+m^{2} k^{2} r^{4}\right) s^{4} \sin ^{2} \theta_{s}} e^{2 i\left(\varphi_{r}-\varphi_{s}\right)}
$$

and

$$
\frac{\beta_{s}^{2}}{\beta_{r}^{2}}=\frac{\left(1+\cos \theta_{s}\right)^{2} \sin ^{2} \theta_{r}}{\left(1+\cos \theta_{r}\right)^{2} \sin ^{2} \theta_{s}} e^{2 i\left(\varphi_{r}-\varphi_{s}\right)} .
$$

The remaining factors require a bit more work:

$$
\begin{aligned}
\left(\zeta_{+}-\beta_{r}\right) & \left(\zeta_{-}-\beta_{r}\right)=s^{2} e^{-i \varphi_{r}}\left(1+\cos \theta_{r}\right)\left(1-i m k r^{2}\right) \\
& \times \frac{\left[\sin \theta_{s}\left(1-\cos \theta_{r}\right) e^{i\left(\varphi_{r}-\varphi_{s}\right)}-\sin \theta_{s}\left(1+\cos \theta_{r}\right) e^{-i\left(\varphi_{r}-\varphi_{s}\right)}+2 \cos \theta_{s} \sin \theta_{r}\right]}{\sin ^{2} \theta_{r}\left(r^{2} \sin \theta_{r} e^{i \varphi_{r}}\left(1-i m k s^{2} \cos \theta_{s}\right)-s^{2} \cos \theta_{s} e^{i \varphi_{s}}\left(1-i m k r^{2} \cos \theta_{r}\right)\right)}
\end{aligned}
$$

and

$$
\begin{aligned}
\left(\zeta_{+}-\beta_{s}\right) & \left(\zeta_{-}-\beta_{s}\right)=r^{2} e^{-i \varphi_{s}}\left(1+\cos \theta_{s}\right)\left(1-i m k s^{2}\right) \\
& \times \frac{\left[\sin \theta_{r}\left(1-\cos \theta_{s}\right) e^{-i\left(\varphi_{r}-\varphi_{s}\right)}-\sin \theta_{r}\left(1+\cos \theta_{s}\right) e^{i\left(\varphi_{r}-\varphi_{s}\right)}+2 \cos \theta_{r} \sin \theta_{s}\right]}{\sin ^{2} \theta_{s}\left(r^{2} \sin \theta_{r} e^{i \varphi_{r}}\left(1-i m k s^{2} \cos \theta_{s}\right)-s^{2} \cos \theta_{s} e^{i \varphi_{s}}\left(1-i m k r^{2} \cos \theta_{r}\right)\right)}
\end{aligned}
$$

We can simplify these expressions by observing that

$$
\begin{aligned}
& \sin \theta_{s}\left(1-\cos \theta_{r}\right) e^{i\left(\varphi_{r}-\varphi_{s}\right)}-\sin \theta_{s}\left(1+\cos \theta_{r}\right) e^{-i\left(\varphi_{r}-\varphi_{s}\right)}+2 \cos \theta_{s} \sin \theta_{r}= \\
& 4\left(\sin \frac{\theta_{r}}{2} \cos \frac{\theta_{s}}{2} e^{i \frac{\varphi_{r}-\varphi_{s}}{2}}-\sin \frac{\theta_{s}}{2} \cos \frac{\theta_{r}}{2} e^{-i \frac{\varphi_{r}-\varphi_{s}}{2}}\right) \\
& \times\left(\cos \frac{\theta_{r}}{2} \cos \frac{\theta_{s}}{2} e^{-i \frac{\varphi_{r}-\varphi_{s}}{2}}+\sin \frac{\theta_{r}}{2} \sin \frac{\theta_{s}}{2} e^{i \frac{\varphi_{r}-\varphi_{s}}{2}}\right),
\end{aligned}
$$

while

$$
\begin{aligned}
\sin \theta_{r}\left(1-\cos \theta_{s}\right) e^{-i\left(\varphi_{r}-\varphi_{s}\right)}-\sin \theta_{r}\left(1+\cos \theta_{s}\right) e^{i\left(\varphi_{r}-\varphi_{s}\right)}+2 \cos \theta_{r} \sin \theta_{s}= \\
4\left(\sin \frac{\theta_{s}}{2} \cos \frac{\theta_{r}}{2} e^{-i \frac{\varphi_{r}-\varphi_{s}}{2}}-\sin \frac{\theta_{r}}{2} \cos \frac{\theta_{s}}{2} e^{i \frac{\varphi_{r}-\varphi_{s}}{2}}\right) \\
\times\left(\cos \frac{\theta_{r}}{2} \cos \frac{\theta_{s}}{2} e^{i \frac{\varphi_{r}-\varphi_{s}}{2}}+\sin \frac{\theta_{r}}{2} \sin \frac{\theta_{s}}{2} e^{-i \frac{\varphi_{r}-\varphi_{s}}{2}}\right) .
\end{aligned}
$$


The common factors cancel in the quotient, so we arrive at the simpler expression

$$
\begin{aligned}
\frac{\left(\zeta_{+}-\beta_{r}\right)\left(\zeta_{-}-\beta_{r}\right)}{\left(\zeta_{+}-\beta_{s}\right)\left(\zeta_{-}-\beta_{s}\right)}= & -\left(\frac{\cos \frac{\theta_{r}}{2} \cos \frac{\theta_{s}}{2} e^{-i \frac{\varphi_{r}-\varphi_{s}}{2}}+\sin \frac{\theta_{r}}{2} \sin \frac{\theta_{s}}{2} e^{i \frac{\varphi_{r}-\varphi_{s}}{2}}}{\cos \frac{\theta_{r}}{2} \cos \frac{\theta_{s}}{2} e^{i \frac{\varphi_{r}-\varphi_{s}}{2}}+\sin \frac{\theta_{r}}{2} \sin \frac{\theta_{s}}{2} e^{-i \frac{\varphi_{r}-\varphi_{s}}{2}}}\right) \\
& \times\left(\frac{s^{2} e^{-i \varphi_{r}}\left(1+\cos \theta_{r}\right) \sin ^{2} \theta_{s}\left(1-i m k r^{2}\right)}{r^{2} e^{-i \varphi_{s}}\left(1+\cos \theta_{s}\right) \sin ^{2} \theta_{r}\left(1-i m k s^{2}\right)}\right) .
\end{aligned}
$$

Looking back at the definitions of $U$ and $\bar{U}$ from (4.2) and (4.3), we see that

$$
\frac{\left(\zeta_{+}-\beta_{r}\right)\left(\zeta_{-}-\beta_{r}\right)}{\left(\zeta_{+}-\beta_{s}\right)\left(\zeta_{-}-\beta_{s}\right)}=-\left(\frac{U}{\bar{U}}\right) e^{i\left(\psi_{r}-\psi_{s}\right)} e^{-i\left(\varphi_{r}-\varphi_{s}\right)}\left(\frac{s^{2}\left(1+\cos \theta_{r}\right) \sin ^{2} \theta_{s}\left(1-i m k r^{2}\right)}{r^{2}\left(1+\cos \theta_{s}\right) \sin ^{2} \theta_{r}\left(1-i m k s^{2}\right)}\right) .
$$

The last expression we need turns out to be

$$
\frac{\left(a_{s}+b_{s} \zeta_{+}\right)\left(a_{s}+b_{s} \zeta_{-}\right)}{\left(a_{r}+b_{r} \zeta_{+}\right)\left(a_{r}+b_{r} \zeta_{-}\right)}=1 .
$$

Combining (6.21), (6.22), (6.28) and (6.29) and replacing the variable $\tau$ by $\psi$ (using (6.3)) we see that (6.20) finally becomes

$$
\mathcal{A}_{+} \mathcal{A}_{-}=\left(\frac{U}{\bar{U}}\right)^{2}
$$

We thus conclude that the functions of $\mathcal{U}, \mathcal{T}$ that reduce to 1 when the points $x_{r}$ and $x_{s}$ are null separated are given by

$$
e^{\mathcal{U}}=\left\{\frac{r^{2}+s^{2}+\sqrt{\Delta}-2 i m k r^{2} s^{2} \mathrm{v}_{2}}{r^{2}+s^{2}-\sqrt{\Delta}-2 i m k r^{2} s^{2} \mathrm{v}_{2}}\right\}^{\frac{1}{2}}\left\{\frac{1-i m k \sqrt{\Delta}+m^{2} k^{2} r^{2} s^{2}\left(2 \mathrm{v}_{2}-1\right)}{1+i m k \sqrt{\Delta}+m^{2} k^{2} r^{2} s^{2}\left(2 \mathrm{v}_{2}-1\right)}\right\}^{\frac{1}{4}\left(1+i \frac{m}{k}\right)}
$$

and

$$
e^{i \mathcal{T}}=\sqrt{\frac{\bar{U}}{U}}
$$

Following [36, we define the functions of null separation as

$$
\mathcal{S}_{ \pm}=1-e^{i \mathcal{T} \mp \mathcal{U}}
$$

Our construction now assures that these functions vanish on the null cones.

\section{The Pedersen Green's function}

We now have functions of the coordinates $x_{r}, x_{s}$ that vanish when these points are on each other's null cones. Associating, as usual, the coordinate $\psi$ with euclidean time, we follow Page's argument that for fixed "spatial" coordinates (in this case, $\vec{x}=(r, \theta, \varphi))$ the Green's function should only have simple poles in $\mathcal{S}_{+}$and $\mathcal{S}_{-}$at $\mathcal{S}_{ \pm}=0$. Restricting to the simplest case, that of conformal coupling, we make the ansatz ${ }^{19}$ :

$$
G_{1}^{(m, k)}=\Phi\left(\vec{x}_{r}, \vec{x}_{s}\right)\left(\frac{1}{\mathcal{S}_{+}}-\frac{1}{\mathcal{S}_{-}}\right)=\Phi\left(\vec{x}_{r}, \vec{x}_{s}\right)\left(\frac{\sinh \mathcal{U}}{\cosh \mathcal{U}-\cos \mathcal{T}}\right)
$$

\footnotetext{
${ }^{19}$ We use the notation $G_{1}^{(m, k)}$ to emphasise that it vanishes as $\left(1-k^{2} r^{2}\right)$ at the boundary, which we anticipate from the known limits (section 4). We can also anticipate that $\Phi$ will diverge as $\sim 1 / \sqrt{\Delta}$ as $\vec{x}_{s}$ is taken to be close to $\vec{x}_{r}$, since $\sinh \mathcal{U} \sim \sqrt{\Delta}$ in this limit. One could turn this argument around, claiming that $G^{(m, k)}$ should not have a pole at $\vec{x}_{r}=\vec{x}_{s}$ if $\psi_{r} \neq \psi_{s}$, and thus try to write a more general ansatz for other masses.
} 
All that remains is to find the function $\Phi\left(\vec{x}_{r}, \vec{x}_{s}\right)$. To do this we can integrate the laplacian (4.1) over a cycle of $\psi(0 \leq \psi \leq 4 \pi)$ to obtain (after converting back to the remaining $\mathrm{v}_{2}$ angular coordinate)

$$
\begin{aligned}
{ }^{(3)} \nabla^{2}=\frac{\left(1-k^{2} r^{2}\right)^{2}}{1+m^{2} r^{2}} & \left(\frac{1}{4}\left(1+m^{2} k^{2} r^{4}\right) \partial_{r r}+\frac{1}{4 r\left(1-k^{2} r^{2}\right)}\left[3+k^{2} r^{2}+7 m^{2} k^{2} r^{4}-3 m^{2} k^{4} r^{6}\right] \partial_{r}\right. \\
+ & \left.\frac{1}{r^{2}}\left[\mathrm{v}_{2}\left(1-\mathrm{v}_{2}\right) \partial_{\mathrm{v}_{2} \mathrm{v}_{2}}+\left(1-2 \mathrm{v}_{2}\right) \partial_{\mathrm{v}_{2}}\right]\right)
\end{aligned}
$$

So for conformal coupling we look for a Green's function ${ }^{(3)} G$ for the laplacian (on some "auxiliary" non-Einstein three-dimensional space)

$$
\left({ }^{(3)} \nabla^{2}+2 k^{2}\right)^{(3)} G=0
$$

where ${ }^{(3)} G$ is related to the Pedersen Green's function we are looking for by

$$
{ }^{(3)} G\left(r, s, \mathrm{v}_{2}\right)=\int_{0}^{4 \pi} \mathrm{d} \psi_{r} G_{1}^{(m, k)}\left(r, s, \mathrm{v}_{1}, \mathrm{v}_{2}\right) .
$$

Fortunately, it turns out that one can easily invert (7.3) to obtain

$$
{ }^{(3)} G\left(r, s, \mathrm{v}_{2}\right)=\frac{\left(1-k^{2} r^{2}\right)\left(1-k^{2} s^{2}\right)}{\sqrt{\Delta}}
$$

where, as before, $\Delta=\left(r^{2}-s^{2}\right)^{2}-4 r^{2} s^{2}\left(\mathrm{v}_{2}-1\right)-4 m^{2} k^{2} r^{4} s^{4} \mathrm{v}_{2}\left(\mathrm{v}_{2}-1\right)$.

The last step is to perform the integration in (7.4). To do that we notice that $\cos \mathcal{T}=$ $\cos \left(\left(\psi_{r}-\psi_{s}\right) / 2+\mathcal{S}\right)$, where $\mathcal{S}=\arctan \left(\cos \frac{1}{2}\left(\theta_{r}-\theta_{s}\right) / \cos \frac{1}{2}\left(\theta_{r}-\theta_{s}\right) \tan \left(\varphi_{r}-\varphi_{s}\right)\right)$ and that (for an arbitrary $X)$

$$
\int_{0}^{4 \pi} \frac{\mathrm{d} t}{a+b \cos (t / 2+X)}=\frac{4 \pi}{\sqrt{a^{2}-b^{2}}} .
$$

In our case $a=\cosh \mathcal{U}$ and $b=1$. So we can compute (7.4) to be:

$$
\int_{0}^{4 \pi} \mathrm{d} \psi_{r} G_{1}^{(m, k)}=4 \pi \Phi\left(\vec{x}_{r}, \vec{x}_{s}\right) \frac{\sinh \mathcal{U}}{\sqrt{\cosh ^{2} \mathcal{U}-1}}=4 \pi \Phi\left(\vec{x}_{r}, \vec{x}_{s}\right) .
$$

Thus $4 \pi \Phi\left(\vec{x}_{r}, \vec{x}_{s}\right)={ }^{(3)} G\left(r, s, \mathrm{v}_{2}\right)$. Introducing the notation

$$
\xi_{ \pm}=\left(r^{2}+s^{2} \pm \sqrt{\Delta}-2 i m k r^{2} s^{2} \mathrm{v}_{2}\right)\left(1 \mp i m k \sqrt{\Delta}+m^{2} k^{2} r^{2} s^{2}\left(2 \mathrm{v}_{2}-1\right)\right)^{\frac{1}{2}\left(1+\frac{i m}{k}\right)}
$$

and

$$
\gamma=\left[\left(1+m^{2} k^{2} r^{4}\right)\left(1+m^{2} k^{2} s^{4}\right)\right]^{\frac{1}{4}\left(1+\frac{i m}{k}\right)} \sqrt{\left(1-i m k r^{2}\right)\left(1-i m k s^{2}\right)},
$$

and absorbing all normalisation factors into the usual coefficient $C$, we conclude that the Green's function for a conformally coupled scalar propagating on QTN is

$$
G_{1}^{(m, k)}\left(r, s, \mathrm{v}_{1}, \mathrm{v}_{2}\right)=C \cdot \frac{\left(1-k^{2} r^{2}\right)\left(1-k^{2} s^{2}\right)}{\sqrt{\Delta}} \frac{\xi_{+}-\xi_{-}}{\xi_{+}+\xi_{-}-2 r s \gamma \mathrm{v}_{1}} .
$$


This result can be verified by direct calculation ${ }^{20}$. It is a real function, symmetric in $x_{r}, x_{s}$. One can check that it has a pole as $x_{s} \rightarrow x_{r}$, as it should, and that it doesn't have a pole as $\vec{x}_{s} \rightarrow \vec{x}_{r}$ if $\psi_{r} \neq \psi_{s}$. Taking the limit of zero cosmological constant $(k \rightarrow 0)$ we see that $\Delta \rightarrow 4 \mathrm{w}^{2}$ (from (4.22) $), \gamma \rightarrow 1$ and

$$
\xi_{ \pm} \longrightarrow\left(r^{2}+s^{2} \pm 2 \mathrm{w}\right) e^{ \pm m^{2} \mathrm{w}}
$$

A brief calculation shows that this indeed gives the Green's function for euclidean Taub-NUT (4.21).

All previous calculations were for the oblate Pedersen metric (3.1). One could perform an analogous calculation for the prolate case, but it is far simpler to analytically continue the parameter $m$ as in (3.6) to obtain the Green's function:

$$
G_{1}^{(\mu, k)}=C \cdot \frac{\left(1-k^{2} r^{2}\right)\left(1-k^{2} s^{2}\right)}{\sqrt{D}} \frac{q_{+}-q_{-}}{q_{+}+q_{-}-2 r s c v_{1}},
$$

where now

$$
q_{ \pm}=\left(r^{2}+s^{2} \pm \sqrt{D}+2 \mu k r^{2} s^{2} \mathrm{v}_{2}\right)\left(1 \pm \mu k \sqrt{D}-\mu^{2} k^{2} r^{2} s^{2}\left(2 \mathrm{v}_{2}-1\right)\right)^{\frac{1}{2}\left(1-\frac{\mu}{k}\right)}
$$

(here $\left.D=\left(r^{2}-s^{2}\right)^{2}-4 r^{2} s^{2}\left(\mathrm{v}_{2}-1\right)+4 \mu^{2} k^{2} r^{4} s^{4} \mathrm{v}_{2}\left(\mathrm{v}_{2}-1\right)\right)$ and

$$
c=\left[\left(1-\mu^{2} k^{2} r^{2}\right)\left(1-\mu^{2} k^{2} s^{2}\right)\right]^{\frac{1}{4}\left(1-\frac{\mu}{k}\right)} \sqrt{\left(1+\mu k r^{2}\right)\left(1+\mu k s^{2}\right)} .
$$

It is easy to verify that $G_{1}^{(\mu, k)}$ reduces to the $G_{1}^{(0, k)}$ and $G_{1}^{(k, k)}$ of section 4 when taking the appropriate limits ( $\mu \rightarrow 0$ and $\mu \rightarrow k$ respectively).

Finally we can use the standard identification between the bulk-to-bulk and bulk-toboundary Green's functions to obtain (in the prolate case) ${ }^{21}$

$$
K_{1}^{(\mu, k)}=\lim _{s \rightarrow 1 / k}\left\{\frac{G_{1}^{(\mu, k)}}{\left(1-k^{2} s^{2}\right)}\right\}=\frac{\left(1-k^{2} r^{2}\right)}{\sqrt{D^{0}}} \frac{q_{+}^{0}-q_{-}^{0}}{q_{+}^{0}+q_{-}^{0}-2 k r c^{0} \mathrm{v}_{1}}
$$

where now

$$
q_{ \pm}^{0}=\left(1+k^{2} r^{2} \pm \sqrt{D^{0}}+2 \mu k r^{2} \mathrm{v}_{2}\right)\left(1 \pm \frac{\mu}{k} \sqrt{D^{0}}-\mu^{2} r^{2}\left(2 \mathrm{v}_{2}-1\right)\right)^{\frac{1}{2}\left(1-\frac{\mu}{k}\right)}
$$

with $D^{0}=\left(1-k^{2} r^{2}\right)^{2}-4 k^{2} r^{2}\left(\mathrm{v}_{2}-1\right)+4 \mu^{2} k^{2} r^{4} \mathrm{v}_{2}\left(\mathrm{v}_{2}-1\right)$ and

$$
c^{0}=\left[\left(1-\mu^{2} k^{2} r^{4}\right)\left(1-\mu^{2} / k^{2}\right)\right]^{\frac{1}{4}\left(1-\frac{\mu}{k}\right)} \sqrt{(1+\mu / k)\left(1+\mu k r^{2}\right)} .
$$

We can check that as $r$ approaches the boundary, $K_{1}^{(\mu, k)} \rightarrow\left(1-k^{2} r^{2}\right)^{2} \delta^{3}(\theta, \varphi, \psi)$. So $K_{1}^{(\mu, k)}$ indeed generalises the $\mathbb{H H}^{1}$ bulk-to-boundary propagator to QTN.

\footnotetext{
${ }^{20}$ To show that $G_{1}^{(m, k)}$ is a Green's function for the laplacian (4.6) with conformal coupling, we find it easier to write it in the form (7.1) and keep the factors of $\cosh \mathcal{U}$ and $\sinh \mathcal{U}$ up to the end of the calculation. So for example if $G_{1}^{(m, k)}=\Phi \cdot F$, with $F=\sinh \mathcal{U} /(\cosh \mathcal{U}-\cos \mathcal{T})$, then $\partial_{r} F=(1-\cos \mathcal{T} \cosh \mathcal{U}) /(\cosh \mathcal{U}-\cos \mathcal{T})^{2} / 2 \cdot \partial_{r} X / X$, where $X=\xi_{+} / \xi_{-}$. This gets rid of the square roots and powers of $1 / 2(1+i m / k)$ that would make the calculation much harder. Note also that since $\left(\nabla^{2}+2 k^{2}\right) \Phi=0$, we need to consider only $\nabla^{2}(\Phi \cdot F)$ with at least one derivative hitting $F$.

${ }^{21} \mathrm{As}$ in section 4 we normalise the bulk-to-boundary propagator to 1 when $r=0$.
} 
Having obtained this result, we could now proceed to define boundary correlation functions by emulating Witten's arguments in [2] or, if we wanted to be careful about their normalisation, by applying the methods of holographic renormalisation. To do that, we would need to convert our results to the Fefferman-Graham coordinate system [28, regulate the action and add boundary counterterms to cancel divergences, as explained (for scalars in a fixed gravitational background) in [16]. (See also [17, Section 5.7 for an illustrative example.) This is currently under investigation $(73])$.

\section{Comments on the boundary CFT}

In this section we indicate what one could learn about the boundary CFT from the construction of the conformal QTN Green's function. We don't provide any new results, just motivation for what should be a much more extensive treatment 73 .

Little is known about conformal field theories in $d=3^{22}$. In the best-known case $\left(\mathrm{AdS}_{4} \times \mathrm{S}^{7}\right)$ where $\mathcal{N}=8$ supersymmetry is preserved, it is believed that the dual CFT is the infrared limit of large $N, \mathcal{N}=8 \mathrm{SYM}$ theory. It is natural to conjecture that the strongly coupled CFT dual to QTN is simply the infrared limit of large $N, d=3$ Yang-Mills theory (or, more precisely, the field theory living on the worldvolume of $N$ M2-branes), but now defined on a squashed $\mathrm{S}^{3}$ rather that the round one. Note that the conformal group of the squashed sphere (i.e. the maximal group preserving the lightcones) seems to be in fact equal to the isometry group $\mathrm{SU}(2) \times \mathrm{U}(1)$.

Another, perhaps more useful, way of looking at the dual theory is as a (non-supersymmetric) deformation of the $\mathcal{N}=8$ theory $^{23}$ : Since the metric deformation from AdS to QTN is a classical background satisfying the boundary conditions, we can interpret it as a source coupling to the dual CFT operator (as is standard in AdS/CFT, e.g. [78]), which for the transverse-traceless mode is the CFT stress-tensor ${ }^{24}$. In this way we could indeed interpret this deformation as a kind of RG-flow, thinking of the field theory as still living on the round sphere (and thus, essentially, on flat space) but with extra terms in the Lagrangian. For the moment, however, we will continue to consider the boundary as a squashed $S^{3}$.

Notice that if one agrees to keep the $S^{7}$ part of the compactification unchanged, the masses of the bulk fields should also not change as one passes from AdS to QTN (since the cosmological constant is the same). However, given that the boundary first becomes negatively curved and then degenerates as we take the limit where QTN gives the Bergman space, it is not clear whether we can simply use the existing AdS/CFT dictionary when relating bulk masses to conformal dimensions of dual operators. Assuming that there is a well-defined correspondence, we examine a few features that we expect to be generic.

The first question we should answer is whether the bulk-to-bulk and bulk-to-boundary propagators we have computed are of any use in "realistic" situations. To rephrase the question, are there any conformally coupled scalars in the Kaluza-Klein reduction of eleven-dimensional supergravity down to $\mathrm{AdS}_{4}$ (and thus presumably also QTN)? Fortunately, as is well known

\footnotetext{
${ }^{22}$ Of course, in flat space, conformal invariance allows us to go quite far. See e.g. $74 \quad 75\left[\begin{array}{ll|l|}76 & 76\end{array}\right.$.

${ }^{23}$ This interpretation was suggested to me by K. Skenderis.

${ }^{24}$ The deformation $h_{\mu \nu}=g_{\mu \nu}^{(\mu, k)}-g_{\mu \nu}^{(0, k)}$, linearised in $\mu$, is not tranverse and traceless, having also longitudinal and trace parts, whose dual operators also have to be added to the CFT lagrangian. It is massless in the AdS background, $\Delta_{L} h_{\mu \nu}+2 \nabla_{(\mu} \nabla^{\rho} h_{\nu) \rho}-\nabla_{(\mu} \nabla_{\nu)} h_{\rho}^{\rho}+6 k^{2} h_{\mu \nu}=0$, so the dual operators will have marginal conformal dimension $\Delta=3$. These terms will, however, break the $\mathrm{SO}(1,4)$ conformal group to its $\mathrm{SU}(2) \times \mathrm{U}(1)$ subgroup.
} 
[79, 80, the answer is positive. (Actually, there are three conformally coupled fields, two scalars and a pseudoscalar.)

The usual AdS/CFT dictionary associates to a bulk scalar field of mass $M$ the conformal dimension of its dual operator through the formula

$$
\Delta_{ \pm}=\frac{d}{2} \pm \sqrt{\frac{d^{2}}{4}+\frac{M^{2}}{k^{2}}} .
$$

What is the dual operator to our scalar field (of $M^{2}=-2 k^{2}$ ) in the (known) case of $\mathrm{AdS}_{4}$ ? To answer this question we make use of the results of [62. They considered the implications for AdS/CFT of the well-known fact [60, 81] that for fields of mass

$$
-\frac{d^{2}}{4}<\frac{M^{2}}{k^{2}}<-\frac{d^{2}}{4}+1
$$

in AdS space, there are two possible quantisations. It is clear that our case $\left(d=3, M^{2}=-2 k^{2}\right)$ falls in this category. As mentioned in Section 4.1, the two possibilities are linked to the existence (in this mass range) of two AdS-invariant solutions $\left(G_{1}^{(0, k)}, G_{2}^{(0, k)}\right)$ to the laplace equation for $\mathrm{HH}^{1}$ (4.8), depending on the boundary conditions we wish to impose. From the boundary CFT perspective, this corresponds to the fact that unitarity allows not only operators of conformal dimension $\Delta_{+}$, as is usual in the AdS/CFT correspondence, but also $\Delta_{-}$. We will briefly review how the correspondence works in this case (see [82, 62, 63] for more details, and also [83] for a more group-theoretical viewpoint).

In the usual $\left(\Delta_{+}\right)$correspondence, a bulk field $\Phi$ is related to its boundary value $\Phi_{0}$ through the bulk-to-boundary propagator $K_{\Delta_{+}}$by [2]:

$$
\Phi(r, \theta, \varphi, \psi)=C \cdot \int \mathrm{d} \Omega^{\prime} K_{\Delta_{+}}^{(0, k)}\left(r, \theta, \varphi, \psi, \theta^{\prime}, \varphi^{\prime}, \psi^{\prime}\right) \Phi_{0}^{r}\left(\theta^{\prime}, \varphi^{\prime}, \psi^{\prime}\right) .
$$

This leads to the regular boundary behaviour $\Phi \rightarrow\left(1-k^{2} r^{2}\right)^{d-\Delta_{+}} \Phi_{0}^{r}$ and, via the standard AdS/CFT formula ${ }^{25}$

$$
e^{-I[\Phi(r, \Omega)]}=\left\langle e^{\int \mathrm{d} \Omega^{\prime} \mathcal{O}_{\Delta_{+}}\left(\Omega^{\prime}\right) \Phi_{0}^{r}\left(\Omega^{\prime}\right)}\right\rangle
$$

(where $\Phi$ satisfies its boundary condition) to correlation functions for the dual boundary operator $\mathcal{O}_{\Delta_{+}}$of conformal dimension $\Delta_{+}{ }^{26}$. As suggested in 62 and further explained in 63, to treat the irregular boundary condition $\Phi \rightarrow\left(1-k^{2} r^{2}\right)^{d-\Delta_{-}} \Phi_{0}^{i}$ (which is achieved through a different bulk-to-boundary propagator $K_{\Delta_{-}}$, using

$$
\left.\Phi(r, \theta, \varphi, \psi)=C \cdot \int \mathrm{d} \Omega^{\prime} K_{\Delta_{-}}^{(0, k)}\left(r, \theta, \varphi, \psi, \theta^{\prime}, \varphi^{\prime}, \psi^{\prime}\right) \Phi_{0}^{i}\left(\theta^{\prime}, \varphi^{\prime}, \psi^{\prime}\right) .\right)
$$

one needs to use a different AdS/CFT formula:

$$
e^{-J[\Phi(r, \Omega)]}=\left\langle e^{\int \mathrm{d} \Omega^{\prime} \mathcal{O}_{\Delta_{-}}\left(\Omega^{\prime}\right) \Phi_{0}^{i}\left(\Omega^{\prime}\right)}\right\rangle .
$$

Here the functional $J$ is related to the usual action functional $I$ by a Legendre transformation. This is then the generator of correlation functions for $\mathcal{O}_{\Delta_{-}}$. Furthermore, in 63], Mück and

\footnotetext{
${ }^{25}$ We use the notation $\Omega$ to abbreviate the boundary coordinates $\theta, \varphi, \psi$.

${ }^{26}$ One should keep in mind that 8.4 relates infinite quantities, and to get correct results one needs to regularise and renormalise by adding counterterms as e.g. in [16].
} 
Viswanathan showed that to calculate higher-point functions of $\mathcal{O}_{\Delta_{-}}$, one needs to define a different bulk-to-bulk propagator $G_{\Delta_{-}}$for $\Phi$, related to the usual one $\left(G_{\Delta_{+}}\right)$in a canonical way. This is the propagator that will give $K_{\Delta_{-}}$in the appropriate limit, $G_{\Delta_{-}} \longrightarrow\left(1-k^{2} r^{2}\right)^{\Delta_{-}} K_{\Delta_{-}}$ as $r \rightarrow 1 / k$.

In our case conformal coupling leads to $\Delta_{+}=2$ and $\Delta_{-}=1$. Looking back at (4.11) and (4.12) it is clear that using $K_{1}^{(0, k)}$ in (8.5) leads to $\Phi \rightarrow\left(1-k^{2} r^{2}\right)^{2} \Phi_{0}^{i}$ as $r$ approaches the boundary, while using $K_{2}^{(0, k)}$ in (8.3) leads to $\Phi \rightarrow\left(1-k^{2} r^{2}\right)^{1} \Phi_{0}^{r}$. Thus we recognise the source $\Phi_{0}^{i}$ associated to $K_{1}^{(0, k)}$ as dual to a boundary operator of conformal dimension $\Delta_{-}=1$, and similarly the source $\Phi_{0}^{r}$ linked to $K_{2}^{(0, k)}$ corresponds to a boundary operator of dimension $\Delta_{+}=2$ (these will be relevant operators in the CFT). Of course for minimal coupling $\left(M^{2}=0\right)$ we simply use the standard prescription (8.3), (8.4) to define a marginal boundary operator $\mathcal{O}_{3}$ with conformal dimension $\Delta_{+}=3$.

What are these boundary operators? The free field content of the dual $N=8$ SYM theory is (assuming one dualises the Yang-Mills field to a scalar, which can be done in the free theory) 8 scalars $X^{i}, i=1, \ldots, 8$ and eight fermions $\lambda_{a}, a=1, \ldots, 8$ (we follow the notation of 84]). The $X^{i}, \lambda_{a}$ have canonical dimension $1 / 2,1$ respectively. In the strong coupling limit where AdS/CFT is expected to apply, we can only really know the spectrum of the chiral operators, which are BPS and thus their dimensions are protected. So we can follow those operators back to weak coupling and express them in terms of the free fields.

The matching of Kaluza-Klein fields to CFT operators can be found in [85, 86, 87, 88, 84, We find that the operator with conformal dimension $\Delta=1$, corresponding to a conformally coupled bulk scalar, is $\mathcal{O}_{1}=\operatorname{Str} X^{i_{1}} X^{i_{2}}$ in terms of free fields ${ }^{27}$. There are two operators of conformal dimension $\Delta=2$, given by $\mathcal{O}_{2}=\operatorname{Str} X^{i_{1}} \cdots X^{i_{4}}$ and $\mathcal{O}_{2}^{\prime}=\operatorname{Str} \lambda_{a_{1}} \lambda_{a_{2}}$. The operator $\mathcal{O}_{2}$ corresponds to a bulk scalar, while $\mathcal{O}_{2}^{\prime}$ corresponds to a bulk pseudoscalar. As for the minimally coupled bulk scalar, it couples to $\mathcal{O}_{3}=\operatorname{Str} X^{i_{1}} \cdots X^{i_{6}}$, and a minimally coupled pseudoscalar to $\mathcal{O}_{3}^{\prime}=\operatorname{Str} \lambda \lambda X X$.

Thus we have seen how one matches some operators of low dimension in the $3 d$ CFT to the corresponding bulk-to-boundary propagators $K_{1}^{(0, k)}, K_{2}^{(0, k)}, K_{3}^{(0, k)}$. Now we want to briefly leave $\mathbb{H H}^{1}$ and look at the more general $K_{1}^{(\mu, k)}$ that we constructed in section 7 . It was obtained as the limit of $\left(1-k^{2} r^{2}\right)^{-1} G_{1}^{(\mu, k)}$, where $G_{1}^{(\mu, k)}$ is the bulk-to-bulk propagator corresponding to conformal coupling. Substituting $K_{1}^{(\mu, k)}$ in (8.5) we obtain a scalar mode satisfying the irregular boundary condition. So it is tempting to identify the dual operator to a conformally coupled scalar on QTN, satisfying the $\Delta_{-}$boundary condition, with the same $\mathcal{O}_{1}$ above, via this bulkto-boundary propagator. However we now lack the protection of supersymmetry, so there is no guarantee that the dimension of $\mathcal{O}_{1}$ will be immune to quantum corrections. All we can say is that there should exist, in the dual $3 d$ QFT on the squashed three-sphere, an operator of conformal dimension $\Delta=1$, that reduces to $\mathcal{O}_{1}$ in the $\mu=0$ limit.

Since we have not found expressions for bulk-to-bulk and bulk-to-boundary propagators for $\Delta>1$, we cannot say much about those cases. However, we can make a comment just by looking at the other relatively well-understood endpoint, corresponding to $\mu=k$. We observe that although we can follow the $K_{1}^{(\mu, k)}$ bulk-to-boundary propagator (and thus the $\Delta=1$ operator) all the way from $\mathbb{H H}^{1}$ to $\widetilde{\mathbb{C P}^{2}}$, the same does not seem to be true for the $K_{2}^{(\mu, k)}$ propagator. Indeed, in the Bergman limit $K_{2}^{(k, k)}$ comes not from the conformally coupled scalar

\footnotetext{
${ }^{27}$ The notation Str denotes the symmetrised trace over the colour indices.
} 
but from the minimal one. So it seems that an operator of $\Delta=3$ on the boundary of $\mathbb{H H}^{1}$ (i.e. minimal coupling in the bulk) will end up as an operator of $\Delta=2$ on the boundary of $\widetilde{\mathbb{C P}^{2}}{ }^{28}$, while the $\Delta=2$ operator at $\mu=0$ somehow vanishes from the spectrum at $\mu=k$ (or somewhere along the way $)^{29}$. The fate of this mode is probably related to the interpretation of the second solution we found for conformal coupling in section 4.2. A better understanding of this issue will require finding the QTN Green's functions for arbitrary coupling [73, and checking their behaviour as the degenerate $\mu=k$ limit is reached. Perhaps the relation [63] between $G_{1}^{(0, k)}$ and $G_{2}^{(0, k)}$ holds also in the QTN case, thus providing an indirect way of finding $G_{2}^{(\mu, k)}$.

\section{Conclusions}

In this article we made a first step towards understanding the $d=3$ conformal field theory dual to quaternionic Taub-NUT, by calculating the bulk-to-bulk and bulk-to-boundary Green's functions for the simplest case, that of a conformally coupled scalar. The next step in our construction would be to actually compute correlation functions for $\mathcal{O}_{1}$ on the Berger sphere using (8.6) supplemented by the methods of holographic renormalisation. We leave this crucial step for the future. (Of course, to do conformal field theory on a $d$-dimensional manifold $X_{d}$ equipped with a given conformal structure, one must sum over the contributions of all $d+1-$ dimensional manifolds with negative curvature that induce this conformal structure on their boundary. In the case of the squashed sphere, there are parameter ranges where, apart from QTN we also have AdS-Taub-Bolt as the "filling in" manifold 21, 22]. So a full understanding of large $-N$ CFT on the squashed 3-sphere would require analogous results for AdS-Taub-Bolt -probably a much more difficult problem since it is not self-dual.)

It will be important to extend our results to the minimally coupled case. One could also envisage generalising to scalars of arbitrary mass, and also to fields of higher spin. QTN admits fermions, but as mentioned we expect the $\mathcal{N}=8$ supersymmetry of the $\mu=0$ case to be broken for $\mu \neq 0^{30}$.

Note that there exist some results for conformally coupled scalar (and spinor) effective actions on the squashed three-sphere [90] and on lens spaces [91, especially for the extreme oblate case. However there does not yet seem to be a direct connection between these weak-coupling results and what could possibly be investigated using AdS/CFT.

QTN is just a special case of a whole class of quaternionic Kähler manifolds with negative cosmological constant constructed by Galicki [42]. Just as QTN reduces to ETN in the Ricciflat limit, there are quaternionic Kähler manifolds that reduce to the multicentre Taub-NUT spaces (see also 92 for an explicit quaternionic extension of double Taub-NUT). The boundary of those spaces is again a squashed three-sphere, but this time with identifications, i.e. a lens space of the form $S^{3} / \mathbb{Z}_{k}$. There also exist higher dimensional versions of AdS-Taub-NUT which could possibly be studied in a similar way 93.

\footnotetext{
${ }^{28}$ In stating this we assume that the standard relation between the fall-off at the boundary and the dual conformal dimension continues to hold. This may not be the case, however, since the authors of [10, using a group-theoretical analysis, do associate the massless bulk mode in the Bergman space to a $\Delta=3$ boundary operator.

${ }^{29}$ Perhaps this is related to the breaking of supersymmetry: The fermions in, say, $\mathcal{O}_{2}^{\prime}$ might now get a mass so we don't see them in the infrared.

${ }^{30}$ However, solutions with NUT charge are not entirely hostile to supersymmetry. See [89] for a discussion of supersymmetric Kerr-Newman-AdS-Taub-NUT solutions in the context of $\mathrm{SO}(2)$ gauged $d=4$ supergravity.
} 
It would be very interesting to find a string or M-theory system that reproduces our model in the supergravity limit, by which we mean a configuration of branes (and, probably, KaluzaKlein monopoles) that gives QTN as part of the near horizon limit. $\mathrm{AdS}_{4}$ can, as we know, be reproduced by a stack of M2 branes in the near horizon limit. The reason the KK monopole solution [94, 95 might be a useful starting point is of course that it is related to ETN, i.e. the Ricci-flat limit of QTN. In the end, one would really like an extension of the techniques of 96 (for instance) to the quaternionic Kähler case. As for the Bergman space, it has made an appearance in the context of untwisting the $\mathrm{AdS}_{5} \times \mathrm{S}^{5}$ solution of IIB supergravity into a $\mathrm{S}^{1} \times \widetilde{\mathbb{C P}^{2}} \times \mathrm{S}^{5}$ solution in type IIA* supergravity, by performing a T-duality in the time direction [97. 98$]^{31}$.

It is clear that many things remain to be done to fully understand holography on QTN. The ultimate goal would be to answer some of the questions posed in the introduction, while in the process learning more about holography through a concrete, non-trivial realisation.

\section{Acknowledgments}

I would like to acknowledge useful discussions with R. Roiban and D. Vaman. I would especially like to thank K. Skenderis for communications and very helpful comments on the manuscript, and also M. Roček for guidance and constant encouragement. I am grateful to the State Scholarships Foundation of Greece for financial support during a large part of this project.

\section{A Pedersen vs. AdS-Taub-NUT}

In this article we make use of the "polar-like" Pedersen metric for quaternionic Taub-NUT. This is because in these coordinates both the AdS and Bergman limits are easy to work with. To gain contact with recent literature on AdS-Taub-NUT 21, 22, 23, 50, we show how to relate the metric used in those references to the Pedersen metric.

Substituting (2.3) in (2.2) we obtain the most commonly used metric for AdS-Taub-NUT:

$$
\mathrm{d} s^{2}=\frac{\mathrm{d} r^{2}}{V(r)}+V(r)[\mathrm{d} \tau+2 n \cos \theta \mathrm{d} \varphi]^{2}+\left(r^{2}-n^{2}\right)\left[\mathrm{d} \theta^{2}+\sin ^{2} \theta \mathrm{d} \varphi^{2}\right]
$$

where

$$
V(r)=\frac{r-n}{r+n}\left[1+k^{2}(r-n)(r+3 n)\right],
$$

and $\tau=2 n \psi, k^{2}=-\Lambda / 3$. In this form, there is a nut at $r=n$.

To see that this is the same as the Pedersen metric, we can make the change

$$
r \rightarrow r+n
$$

(transporting the nut to $r=0$ ), followed by the transformation ${ }^{32}$

$$
r=\frac{\rho^{2} /(2 n)}{1-k^{2} \rho^{2}} .
$$

\footnotetext{
${ }^{31}$ It is worth noting that this is a BPS solution (being related to one by T-duality), although at first sight supersymmetry seems to be lost. As explained in [97, the full string theory knows about supersymmetry, but the supergravity limit doesn't exhibit it.

${ }^{32}$ This is a slight adaptation of eq. (2.8) in 22.
} 
Then, identifying $\mu^{2} / k^{2}=1-1 /\left(4 k^{2} n^{2}\right)$ (and relabelling $\rho \rightarrow r$ ) brings the metric to the prolate form (3.7). We see that the Ricci-flat boundary case $\left(\mu^{2}=3 / 4 k^{2}\right)$ corresponds to a nut charge $n=1 / k$, while to reach the Bergman limit $\left(\mu^{2}=k^{2}\right)$ we need to take $n \rightarrow \infty$. At $n=1 /(2 k)$ we recover AdS, and for $n<1 /(2 k)$ we find ourselves in the oblate case.

Taking $k=0$ brings us to the relation between the more commonly used metric for euclidean Taub-NUT (A.1) with $V(r)=(r-n) /(r+n))$ to the one we use in section 3.1, and relates the nut charges of these two metrics.

\section{B Coordinate systems on hyperbolic space}

In the AdS/CFT correspondence, it is usually convenient to write the metric for euclidean AdS in Poincaré (upper half plane) coordinates. Then the conformal flatness of the boundary metric is obvious. In our case, since we are interested in deforming away from the conformally flat case, it is more useful to exhibit the $\mathrm{SU}(2) \times \mathrm{SU}(2)$ symmetry of the boundary by using polartype coordinates for hyperbolic space. Then it is clear how this bi-invariant case arises as a special case of the squashed $\mathrm{SU}(2) \times \mathrm{U}(1)$ metric. Since the bulk of the AdS/CFT literature on propagators uses the Poincaré parametrisation, we give a short explanation of our coordinates and the form of the propagators.

We consider $\mathbb{H H}^{1}$ as the hyperboloid embedded in $\mathbb{R}^{1,4}$ :

$$
X_{0}^{2}-X_{1}^{2}-X_{2}^{2}-X_{3}^{2}-X_{4}^{2}=\frac{1}{k^{2}},
$$

where $1 / k^{2}$ is the radius of curvature. To obtain the Poincaré parametrisation we solve (B.1) by:

$$
\begin{aligned}
& X_{0}=\frac{y}{2}\left(1+\frac{1}{y^{2}}\left(x^{2}+\frac{1}{k^{2}}\right)\right), \\
& X_{a}=\frac{x_{a}}{k y}, \quad a=1,2,3, \\
& X_{4}=\frac{y}{2}\left(1+\frac{1}{y^{2}}\left(x^{2}-\frac{1}{k^{2}}\right)\right),
\end{aligned}
$$

where $x^{2}=\sum_{a=1}^{3} x_{a}^{2}$ (see e.g. 4]). This gives the metric

$$
\mathrm{d} s^{2}=-\mathrm{d} X_{0}^{2}+\sum_{i=1}^{4} \mathrm{~d} X_{i}^{2}=\frac{1}{k^{2} y^{2}}\left(\mathrm{~d} y^{2}+\mathrm{d} x_{1}^{2}+\mathrm{d} x_{2}^{2}+\mathrm{d} x_{3}^{2}\right)
$$

In this parametrisation the boundary is at $y=0$.

On the other hand, to obtain the "polar/stereographic" parametrisation of (3.12) we solve (B.1) as follows (e.g. 99]):

$$
\begin{aligned}
X_{0} & =\frac{1}{k} \frac{1+k^{2} w^{2}}{1-k^{2} w^{2}}, \\
X_{i} & =\frac{2 w_{i}}{1-k^{2} w^{2}} \quad, \quad i=1,2,3,4
\end{aligned}
$$


where here $w^{2}=\sum_{i=1}^{4} w_{i}^{2}$. Then we find

$$
\mathrm{d} s^{2}=-\mathrm{d} X_{0}^{2}+\sum_{i=1}^{4} \mathrm{~d} X_{i}^{2}=\frac{4}{1-k^{2} w^{2}}\left(\mathrm{~d} w_{1}^{2}+\mathrm{d} w_{2}^{2}+\mathrm{d} w_{3}^{2}+\mathrm{d} w_{4}^{2}\right)
$$

First we convert to the complex coordinates $z_{1}=w_{1}+i w_{2}, z_{2}=w_{3}+i w_{4}$ to obtain

$$
\mathrm{d} s^{2}=\frac{4}{1-k^{2}\left(z_{1} \bar{z}_{1}+z_{2} \bar{z}_{2}\right)}\left(\mathrm{d} z_{1} \mathrm{~d} \bar{z}_{1}+\mathrm{d} z_{2} \mathrm{~d} \bar{z}_{2}\right)
$$

and finally we convert to polar coordinates using

$$
z_{1}=r \cos \frac{\theta_{r}}{2} e^{\frac{i}{2}\left(\psi_{r}+\varphi_{r}\right)}, z_{2}=r \sin \frac{\theta_{r}}{2} e^{\frac{i}{2}\left(\psi_{r}-\varphi_{r}\right)}
$$

to obtain the metric in (3.12):

$$
\mathrm{d} s^{2}=\frac{4}{\left(1-k^{2} r^{2}\right)^{2}}\left[\mathrm{~d} r^{2}+r^{2}\left(\sigma_{1}^{2}+\sigma_{2}^{2}+\sigma_{3}^{2}\right)\right] .
$$

Now the boundary is at $r=1 / k$.

We are interested in the expression for the chordal distance in the two coordinate systems. In Poincaré coordinates we have the expression (e.g. [58]):

$$
u=\frac{\left(y-y^{\prime}\right)^{2}-\left(\vec{x}-\vec{x}^{\prime}\right)^{2}}{2 y y^{\prime}},\left(\vec{x}-\vec{x}^{\prime}\right)^{2}=\sum_{a=1}^{3}\left(x_{a}-x_{a}^{\prime}\right)^{2} .
$$

First we convert this to the homogeneous $X_{0}, \ldots, X_{4}$ coordinates using

$$
y=\frac{1}{k^{2}\left(X_{0}-X_{4}\right)}, x_{a}=k y X_{a}, a=1,2,3
$$

to obtain, as expected:

$$
u=\left(k^{2}\left(X_{0} X_{0}^{\prime}-\sum_{i=1}^{4} X_{i} X_{i}^{\prime}\right)-1\right) .
$$

Now we convert to the $w_{i}$ coordinates using (B.4):

$$
u=\frac{2 k^{2}\left(w^{2}+w^{\prime 2}-2 \sum_{i=1}^{4} w_{i} w_{i}^{\prime}\right)}{\left(1-k^{2} w^{2}\right)\left(1-k^{2} w^{\prime 2}\right)} .
$$

Finally we convert to polar coordinates using (B.6) and (B.7) (setting $z_{1}^{\prime}=s \cos \left(\theta_{s} / 2\right) e^{\frac{i}{2}\left(\psi_{s}+\varphi_{s}\right)}$ and so forth) to obtain the chordal distance for hyperbolic space:

$$
u^{(\mu=0, k)}=2 k^{2} \frac{r^{2}+s^{2}-r s \mathrm{v}_{1}}{\left(1-k^{2} r^{2}\right)\left(1-k^{2} s^{2}\right)} .
$$

In this way we have recovered the expression in Section 4.1. 


\section{References}

[1] J. Maldacena, The large $N$ limit of superconformal field theories and supergravity, Adv. Theor. Math. Phys. 2 (1998) 231, hep-th/9711200.

[2] E. Witten, Anti-de Sitter space and holography, Adv. Theor. Math. Phys. 2 (1998) 253, hep-th/9802150.

[3] S. S. Gubser, I. R. Klebanov, and A. M. Polyakov, Gauge theory correlators from non-critical string theory, Phys. Lett. B 428 (1998) 105, hep-th/9802109.

[4] O. Aharony, S. Gubser, J. Maldacena, H. Ooguri, and Y. Oz, Large N field theories, string theory and gravity, Phys. Rep. 323 (2000) 183, hep-th/9905111.

[5] E. Witten, Anti-de Sitter space, thermal phase transition, and confinement in gauge theories, Adv. Math. Phys. 2 (1998) 505, hep-th/9803131.

[6] H. J. Boonstra, K. Skenderis, and P. K. Townsend, The domain-wall/QFT correspondence, J. High Energy Phys. 01 (1999) 03, hep-th/9807137.

[7] R. G. Cai and N. Ohta, Surface counterterms and boundary stress-energy tensors for asymptotically non-anti-de Sitter spaces, Phys. Rev. D 62 (2000) 024006, hep-th/9912013.

[8] M. Cvetič, H. Lü, and C. N. Pope, Space-times of boosted p-branes and CFT in the infinite momentum frame, Nucl. Phys. B 545 (1999) 309, hep-th/9810123.

[9] D. Brecher, A. Chamblin, and H. S. Reall, AdS/CFT in the infinite momentum frame, Nucl. Phys. B 607 (2001) 155, hep-th/0012076.

[10] R. Britto-Pacumio, A. Strominger, and A. Volovich, Holography for coset spaces, J. High Energy Phys. 11 (1999) 013, hep-th/9905211.

[11] A. Strominger, The dS/CFT correspondence, J. High Energy Phys. 10 (2001) 034, hep-th/0106113.

[12] G. T. Horowitz and D. Marolf, A new approach to string cosmology, J. High Energy Phys. 07 (1998) 014, hep-th/9805207.

[13] Y.-H. Gao, AdS/CFT correspondence and quotient space geometry, J. High Energy Phys. 04 (1999) 05, hep-th/9903080.

[14] M. Taylor-Robinson, "Holography for degenerate boundaries." hep-th/0001177, 2000.

[15] M. Henningson and K. Skenderis, The holographic Weyl anomaly, J. High Energy Phys. 07 (1998) 023, hep-th/9806087.

[16] S. de Haro, K. Skenderis, and S. N. Solodukhin, Holographic reconstruction of spacetime and renormalisation in the AdS/CFT correspondence, Commun. Math. Phys. 217 (2001) 595, hep-th/0002230. 
[17] K. Skenderis, Lecture notes on holographic renormalization, Class. Quant. Grav. 19 (2002) 5849, hep-th/0209067.

[18] K. Skenderis, Asymptotically anti-de Sitter spacetimes and their stress energy tensor, Int. J. Mod. Phys. A 16 (2001) 740, hep-th/0010138.

[19] M. Bianchi, D. Z. Freedman, and K. Skenderis, How to go with an RG flow, J. High Energy Phys. 08 (2001) 041, hep-th/0105276.

[20] M. Bianchi, D. Z. Freedman, and K. Skenderis, Holographic renormalization, Nucl. Phys. B 631 (2002) 159, hep-th/0112119.

[21] S. W. Hawking, C. J. Hunter, and D. N. Page, NUT charge, anti-de Sitter space, and entropy, Phys. Rev. D. 59 (1999) 044033, hep-th/9809035.

[22] A. Chamblin, R. Emparan, C. V. Johnson, and R. C. Myers, Large $N$ phases, gravitational instantons, and the nuts and bolts of AdS holography, Phys. Rev. D 59 (1999) 064010, hep-th/9808177.

[23] R. Emparan, C. V. Johnson, and R. C. Myers, Surface terms as counterterms in the AdS/CFT correspondence, Phys. Rev. D 60 (1999) 104001, hep-th/9903238.

[24] R. B. Mann, Misner string entropy, Phys. Rev. D 60 (1999) 104047, hep-th/9903229.

[25] H. Pedersen, Einstein metrics, spinning top motions and monopoles, Math. Ann. 274 (1986) 35.

[26] C. R. LeBrun, $\mathcal{H}$-space with a cosmological constant, Proc. R. Soc. Lond. A 380 (1982) 171.

[27] C. R. Graham and J. M. Lee, Einstein metrics with prescribed conformal infinity on the ball, Adv. Math. 87 (1991) 186.

[28] C. Fefferman and C. R. Graham, Conformal invariants, in Élie Cartan et les mathématiques d'aujourd'hui, Astérisque hors série (1985) 95.

[29] K. Landsteiner and E. Lopez, Probing the strong coupling limit of large N SYM on curved backgrounds, J. High Energy Phys. 09 (1999) 006, hep-th/9908010.

[30] K. Koyama and J. Soda, Strongly coupled CFT in FRW universe from AdS/CFT correspondence, J. High Energy Phys. 05 (2001) 027, hep-th/0101164.

[31] G. L. Cardoso and D. Lüst, The holographic RG flow in a field theory on a curved background, J. High Energy Phys. 09 (2002) 028, hep-th/0207024.

[32] M. J. Duff, B. E. W. Nilsson, and C. N. Pope, Kaluza-Klein supergravity, Phys. Rept. 130 (1986) 1.

[33] N. Seiberg and E. Witten, The D1/D5 system and singular CFT, J. High Energy Phys. 04 (1999) 017, hep-th/9903224. 
[34] E. Witten and S.-T. Yau, Connectedness of the boundary in the AdS/CFT correspondence, Adv. Theor. Math. Phys. 3 (1999) 1635, hep-th/9910245.

[35] B. McInnes, Topologically induced instability in string theory, J. High Energy Phys. 03 (2001) 031, hep-th/0101136.

[36] D. N. Page, Green's functions for gravitational multi-instantons, Phys. Lett. B 85 (1979) 369.

[37] S. Ishihara, Quaternion Kählerian manifolds, J. Diff. Geom. 9 (1974) 483.

[38] S. Salamon, Quaternionic Kähler manifolds, Invent. Math 67 (1982) 143.

[39] E. D'Hoker and D. Z. Freedman, "Supersymmetric gauge theories and the AdS/CFT correspondence (TASI 2001 lecture notes)." hep-th/0201253.

[40] T. Eguchi, P. B. Gilkey, and A. J. Hanson, Gravitation, gauge theories and differential geometry, Phys. Rept. 66 (1980) 213.

[41] K. Galicki, New matter couplings in $N=2$ supergravity, Nucl. Phys. B 289 (1987) 573.

[42] K. Galicki, Multi-centre metrics with negative cosmological constant, Class. Quant. Grav. 8 (1991) 1529.

[43] K. Galicki, Quaternionic Kähler and hyper-Kähler non-linear $\sigma$-models, Nucl. Phys. B 271 (1986) 402.

[44] K. Galicki, A generalisation of the momentum mapping construction for quaternionic Kähler manifolds, Commun. Math. Phys. 108 (1987) 117.

[45] K. Galicki and H. B. Lawson, Quaternionic reduction and quaternionic orbifolds, Math. Ann. 282 (1988) 1.

[46] E. Ivanov and G. Valent, Harmonic space construction of the quaternionic Taub-NUT metric, Class. Quant. Grav. 16 (1999) 1039, hep-th/9810005.

[47] A. Galperin, E. Ivanov, S. Kalitzin, V. Ogievetsky, and E. Sokatchev, Unconstrained $N=2$ matter, Yang-Mills and supergravity theories in harmonic superspace, Class. Quant. Grav. 1 (1984) 469.

[48] A. Galperin, E. Ivanov, and O. Ogievetsky, Harmonic space and quaternionic manifolds, Ann. Phys. 230 (1994) 201, hep-th/9212155.

[49] J. Bagger and E. Witten, Matter couplings in $N=2$ supergravity, Nucl. Phys. B 222 (1983) 1.

[50] K. Behrndt and G. Dall' Agata, Vacua of $\mathcal{N}=2$ gauged supergravity derived from non-homogeneous quaternionic spaces, Nucl. Phys. B 627 (2001) 357, hep-th/0112136.

[51] V. Balasubramanian and P. Kraus, A stress tensor for anti-de Sitter gravity, Commun. Math. Phys. 208 (1999) 413, hep-th/9902121. 
[52] N. J. Hitchin, Twistor spaces, Einstein manifolds and isomonodromic deformations, J. Diff. Geom. 42 (1995) 30.

[53] S. W. Hawking, Gravitational instantons, Phys. Lett. A 60 (1977) 81.

[54] C. P. Burgess and C. A. Lütken, Propagators and effective potentials in anti-de-Sitter space, Phys. Lett. B 153 (1985) 137.

[55] W. Mück and K. S. Viswanathan, Conformal field theory correlators from classical scalar field theory on $A d S_{d+1}$, Phys. Rev. D 58 (1998) 041901, hep-th/9804035.

[56] D. Z. Freedman, S. D. Mathur, A. Matusis, and L. Rastelli, Correlation functions in the $C F T_{d} / A d S_{d+1}$ correspondence, Nucl. Phys. B 546 (1999) 96, hep-th/9804058.

[57] E. D'Hoker, D. Z. Freedman, S. D. Mathur, A. Matusis, and L. Rastelli, Graviton and gauge boson propagators in AdS $S_{d+1}$, Nucl. Phys. B 562 (1999) 330, hep-th/9902042.

[58] E. D'Hoker, D. Z. Freedman, S. D. Mathur, A. Matusis, and L. Rastelli, Graviton exchange and complete 4-point functions in the AdS/CFT correspondence, Nucl. Phys. B 562 (1999) 353, hep-th/9903196.

[59] E. D'Hoker, D. Z. Freedman, and L. Rastelli, AdS/CFT 4-point functions: How to succeed at z-integrals without really trying, Nucl. Phys. B 562 (1999) 395, hep-th/9905049.

[60] P. Breitenlohner and D. Z. Freedman, Stability in gauged extended supergravity, Ann. Phys. (NY) 144 (1982) 249.

[61] S. B. Giddings, The boundary S-matrix and the AdS to CFT dictionary, Phys. Rev. Lett. 83 (1999) 2707, hep-th/9903048.

[62] I. R. Klebanov and E. Witten, AdS/CFT correspondence and symmetry breaking, Nucl. Phys. B 556 (1999) 89, hep-th/9905104.

[63] W. Mück and K. S. Viswanathan, Regular and irregular boundary conditions in the AdS/CFT correspondence, Phys. Rev. D 60 (1999) 081901, hep-th/9906155.

[64] N. P. Warner, Green functions on CP ${ }^{n}$, Proc. Roy. Soc. Lond. A 383 (1982) 207.

[65] G. W. Gibbons and S. W. Hawking, Classification of gravitational instanton symmetries, Commun. Math. Phys. 66 (1979) 291.

[66] N. J. Hitchin, Monopoles and geodesics, Commun. Math. Phys. 83 (1982) 579.

[67] P. E. Jones and K. P. Tod, Minitwistor spaces and Einstein-Weyl spaces, Class. Quantum Grav. 2 (1985) 565.

[68] R. Penrose, Nonlinear gravitons and curved twistor theory, Gen. Rel. Grav. 7 (1976) 31.

[69] M. F. Atiyah, N. J. Hitchin, and I. M. Singer, Self-duality in four-dimensional riemannian geometry, Proc. Roy. Soc. Lond. A 362 (1978) 425.

[70] N. J. Hitchin, Polygons and gravitons, Math. Proc. Camb. Phil. Soc. 85 (1979) 465. 
[71] R. S. Ward, Self-dual space-times with cosmological constant, Commun. Math. Phys. 78 (1980) 1.

[72] N. J. Hitchin, Complex manifolds and Einstein's equations, in Lecture Notes in Mathematics, vol. 970, p. 73. Springer-Verlag, 1982.

[73] K. Zoubos (in preparation).

[74] V. K. Dobrev, G. Mack, V. B. Petkova, S. G. Petrova, and I. T. Todorov, Harmonic Analysis on the $n$-Dimensional Lorentz Group and its Application to Conformal Quantum Field Theory, vol. 63 of Lecture Notes in Physics. Springer-Verlag, 1977.

[75] H. Osborn and A. Petkos, Implications of conformal invariance in field theories for general dimensions, Ann. Phys. (NY) 231 (1994) 311, hep-th/9307010.

[76] E. S. Fradkin and M. Ya. Palchik, Conformal Quantum Field Theory in D-Dimensions. Kluwer, 1996.

[77] E. S. Fradkin and M. Ya. Palchik, New developments in D-dimensional conformal quantum field theory, Phys. Rept. 300 (1998) 1.

[78] V. Balasubramanian, P. Kraus, A. Lawrence, and S. P. Trivedi, Holographic probes of anti-de Sitter spacetimes, Phys. Rev. D 59 (1999) 104021, hep-th/9808017.

[79] B. Biran, A. Casher, F. Englert, M. Rooman, and P. Spindel, The fluctuating seven-sphere in eleven-dimensional supergravity, Phys. Lett. B 134 (1984) 179.

[80] L. Castellani, R. D. Auria, P. Fré, K. Pilch, and P. van Nieuwenhuizen, The bosonic mass formula for Freund-Rubin solutions of D=11 supergravity on general coset manifolds, Class. Quantum Grav. 1 (1984) 339.

[81] P. Breitenlohner and D. Z. Freedman, Positive energy in Anti-de Sitter backgrounds and gauged extended supergravity, Phys. Lett. B 115 (1982) 197.

[82] V. Balasubramanian, P. Kraus, and A. Lawrence, Bulk versus boundary dynamics in anti-de Sitter spacetime, Phys. Rev. D 59 (1999) 046003, hep-th/9805171.

[83] V. K. Dobrev, Intertwining operator realization of the AdS/CFT correspondence, Nucl. Phys. B 553 (1999) 559, hep-th/9812194.

[84] E. D'Hoker and B. Pioline, Near-extremal correlators and generalized consistent truncation for $A d S_{4 \mid 7} \times S^{7 \mid 4}$, J. High Energy Phys. 07 (2000) 021, hep-th/0006103.

[85] E. Halyo, Supergravity on $A d S_{4 / 7} \times S^{7 / 4}$ and M branes, J. High Energy Phys. 04 (1998) 011, hep-th/9803077.

[86] O. Aharony, Y. Oz, and Z. Yin, $M$ theory on $A d S_{p} \times S^{11-p}$ and superconformal field theories, Phys. Lett. B 430 (1998) 87, hep-th/9803051.

[87] S. Minwalla, Particles on $A d S_{4 / 7}$ and primary operators on $M_{2 / 5}$ brane worldvolumes, $J$. High Energy Phys. 10 (1998) 002, hep-th/9803053. 
[88] R. Entin and J. Gomis, Spectrum of chiral operators in strongly coupled gauge theories, Phys. Rev. D 58 (1998) 105008, hep-th/9804060.

[89] N. Alonso-Alberca, P. Meessen, and T. Ortin, Supersymmetry of topological Kerr-Newman-Taub-NUT-AdS spacetimes, Class. Quant. Grav. 17 (2000) 2783, hep-th/0003071.

[90] J. S. Dowker, Effective actions on the squashed 3-sphere, Class. Quant. Grav. 16 (1999) 1937, hep-th/9812202.

[91] M. De Francia, K. Kirsten, and J. S. Dowker, Effective actions on squashed lens-spaces, Class. Quant. Grav. 18 (2001) 955, hep-th/0008059.

[92] P.-Y. Casteill, E. Ivanov, and G. Valent, U(1) $\times \mathrm{U}(1)$ quaternionic metrics from harmonic superspace, Nucl. Phys. B 627 (2002) 403, hep-th/0110280.

[93] A. M. Awad and A. Chamblin, A bestiary of higher dimensional Taub-NUT-AdS spacetimes, Class. Quant. Grav. 19 (2002) 2051, hep-th/0012240.

[94] R. Sorkin, Kaluza-Klein monopole, Phys. Rev. Lett. 51 (1983) 87.

[95] D. J. Gross and M. J. Perry, Magnetic monopoles in Kaluza-Klein theories, Nucl. Phys. B 226 (1983) 29.

[96] J. P. Gauntlett, G. W. Gibbons, G. Papadopoulos, and P. K. Townsend, Hyper-Kähler manifolds and multiply intersecting branes, Nucl. Phys. B 500 (1997) 133, hep-th/9702202.

[97] C. N. Pope, A. Sadrzadeh, and S. R. Scuro, Timelike Hopf duality and type IIA* string solutions, Class. Quant. Grav. 17 (2000) 623, hep-th/9905161.

[98] A. Kehagias and J. G. Russo, Hyperbolic spaces in string and M-theory, J. High Energy Phys. 07 (2000) 027, hep-th/0003281.

[99] J. L. Petersen, Introduction to the Maldacena conjecture on AdS/CFT, Int. J. Mod. Phys. A 14 (1999) 3597, hep-th/9902131. 